\title{
Wilson surfaces in M5-branes
}

\author{
Prarit Agarwal, ${ }^{a}$ Joonho Kim, ${ }^{b}$ Seok Kim ${ }^{a}$ and Antonio Sciarappa ${ }^{b}$ \\ ${ }^{a}$ Department of Physics and Astronomy $\&$ Center for Theoretical Physics, \\ Seoul National University, 1 Gwanak-ro, Gwanak-gu, Seoul, 08826 Korea \\ ${ }^{b}$ School of Physics, Korea Institute for Advanced Study, \\ 85 Hoegi-ro, Dongdaemun-gu, Seoul, 02455 Korea \\ E-mail: agarwalprarit@gmail.com, joonhokim@kias.re.kr, \\ skim@phya.snu.ac.kr, asciara@kias.re.kr
}

ABStRACT: We study Wilson surface defects in $6 \mathrm{~d} \mathcal{N}=(2,0)$ superconformal field theories, engineered by semi-infinite M2-branes intersecting M5-branes. Two independent approaches are used to obtain the Wilson surface observables on the $T^{2}$ inside $\Omega$-deformed $\mathbf{R}^{4} \times T^{2}$. One approach is to compute $5 \mathrm{~d} \mathcal{N}=1^{*}$ instanton partition functions in the presence of Wilson lines, where the instanton corrections capture the 6d Kaluza-Klein momentum modes. The other approach is to study the elliptic genera of $2 \mathrm{~d} \mathcal{N}=(0,4)$ gauge theories, which we propose as describing $6 \mathrm{~d}$ self-dual strings in the presence of Wilson surface defects. We make a detailed comparison between these two independent computations, which precisely agree for Wilson surfaces in minuscule representations; for non-minuscule representations instead we only find partial agreement, due to technical problems which we comment about.

Keywords: Brane Dynamics in Gauge Theories, Duality in Gauge Field Theories, Field Theories in Higher Dimensions, Wilson, 't Hooft and Polyakov loops

ARXiv EPrint: 1804.09932 


\section{Contents}

1 Introduction 1

2 Line operators in $5 \mathrm{~d} \mathcal{N}=1^{*}$ gauge theory 2

2.1 Review of ADHM quantum mechanics 3

$\begin{array}{ll}2.2 & \text { ADHM index with line operators }\end{array}$

$\begin{array}{ll}2.3 \text { The Wilson loop observable } & 11\end{array}$

3 Wilson surfaces in $6 \mathrm{~d} \mathcal{N}=(2,0)$ SCFTs $\quad 16$

$\begin{array}{lll}3.1 & \text { Gauge theories on self-dual strings } & 16\end{array}$

$\begin{array}{ll}3.2 & \text { Elliptic genera with Wilson surfaces }\end{array}$

$\begin{array}{lll}3.3 & \text { Relation to 5d Wilson loops } & 23\end{array}$

$\begin{array}{ll}3.3 .1 & \text { qq-characters and } y \text { observables }\end{array}$

4 Concluding remarks $\quad 28$

\section{Introduction}

Wilson loop operators in gauge theories are among the most important observables one can study. They correspond to the phase factor induced from the parallel transport of a charged probe particle around a loop. These non-local and gauge invariant observables can be used to formulate the classical gauge theory itself, encoding some important aspects of the strongly-coupled gauge dynamics. For example the expectation value of a Wilson loop, which consists of a pair of semi-infinite straight lines, captures the interaction potential between a quark and an antiquark, becoming an order parameter for quark confinement.

In a $6 \mathrm{~d}$ superconformal field theory (SCFT) which contains the two-form potential $B_{\mu \nu}$ and the associated supersymmetric strings, the Wilson loop operator of gauge theories can be generalized to the phase factor induced from moving a charged probe string along a spacetime trajectory given by a $2 \mathrm{~d}$ surface $\Sigma$. This is the Wilson surface operator [1] which we will study throughout this paper. It can formally be expressed in the following form:

$$
\mathcal{W}_{\mathbf{R}}[\Sigma]=\operatorname{Tr}_{\mathbf{R}}\left(\mathcal{P} \exp i \int_{\Sigma} d \sigma^{\mu \nu}\left(B_{\mu \nu}+\cdots\right)\right)
$$

where $\cdots$ must be determined from supersymmetric completion for unbroken generators. As we lack a formalism for $6 \mathrm{~d}$ SCFTs with a non-Abelian self-dual tensor multiplet, the precise meaning of 'representation' can only be clarified through string theory realization of the Wilson surface defects. For example, an $n$-wound membrane ending on $\Sigma$ realizes the Wilson surface in rank- $n$ symmetric representation, while $n$ membranes ending on $\Sigma$ engineers the rank- $n$ antisymmetric Wilson surface [2]. 
We will particularly focus on the $6 \mathrm{~d} \mathcal{N}=(2,0) \mathrm{SCFT}$ of $A_{N-1}$-type that describes the worldvolume dynamics of $N$ parallel M5-branes. It contains tensionless BPS strings that are induced from open M2-branes suspended between M5-brane pairs [3, 4]. We will consider the tensor branch of $(2,0)$ SCFT defined on $\mathbf{R}^{4} \times T^{2}$, i.e. the configuration in which all M5-branes are separated from each other. Such a separation gives the BPS string a nonzero tension proportional to the distance between M5-branes. Alongside these relatively light strings, we will also introduce heavy probe strings induced from semi-infinite M2branes ending on M5-branes, which engineer the Wilson surface defect. This is a $\frac{1}{2}$-BPS defect preserving only 8 Poincaré supercharges.

The expectation value and operator product expansion of Wilson surface operators in the fundamental representation was studied using holographic techniques in [5-7]. This was generalized to the case of large-rank antisymmetric and symmetric representations in $[2,8-$ 10]. The field-theoretic calculations of the Wilson surface defects were made through $5 \mathrm{~d}$ SYM description of $6 \mathrm{~d}(2,0)$ SCFTs on $S^{5} \times S^{1}$ [11-13] and $\Omega$-deformed $\mathbf{R}^{4} \times T^{2}[14,15]$. An agreement between two computations was found in [16] for the large-rank symmetric and antisymmetric Wilson surfaces on $T^{2} \subset S^{5} \times S^{1}$.

In this paper, we extend the field-theoretic computation to the case of the Wilson surface observables wrapping $T^{2}$ inside $\Omega$-deformed $\mathbf{R}^{4} \times T^{2}[14,15]$ using two independent approaches. One way is to compute $5 \mathrm{~d} \mathcal{N}=1^{*}$ instanton partition function in the presence of Wilson loops, where the instanton corrections capture the 6d Kaluza-Klein momentum modes. For this purpose, we consider the ADHM quantum mechanics of SYM instantons with Wilson loops [17] and compute their Witten indices. Specifically for minuscule representations, which were already studied in [14, 15], the ADHM indices take a simple form so that one can easily extract the instanton corrected Wilson loop observables out of them. We also explain the general structure of the ADHM indices and identify the Wilson loops in various other representations beyond the minuscule ones. This $5 \mathrm{~d}$ approach will be discussed in section 2 . The other way is to consider the elliptic genera of $2 \mathrm{~d} \mathcal{N}=(0,4)$ quiver gauge theories that describe $6 \mathrm{~d}$ BPS strings in the presence of a Wilson surface defect. We describe these quivers in section 3 , thereby generalizing $[18,19]$. In section 3.3 , we compare the results from the above two independent computations and find precise agreement for Wilson surfaces in minuscule representations; however for non-minuscule representations there seems to be only a partial match. This is due to technical problems on which we comment at the end of the section.

\section{Line operators in $5 \mathrm{~d} \mathcal{N}=1^{*}$ gauge theory}

In this section, we shall study $\frac{1}{2}$-BPS line defects in $5 \mathrm{~d} \mathcal{N}=1^{*} \mathrm{U}(N)$ gauge theory. The string theory realization of $5 \mathrm{~d}$ maximal SYM is a stack of $N$ coincident D4-branes, which we assume to fill the $x^{0}, \cdots, x^{4}$ directions. It preserves $\mathrm{SO}(1,4)$ Lorentz symmetry and $\mathrm{SO}(5)_{R} \mathrm{R}$-symmetry that rotates the transverse $x^{5}, \cdots, x^{9}$ directions. We consider a particular configuration where all D4-branes are separated from one another along the $x^{9}$ axis. This corresponds to the Coulomb branch of 5d maximal SYM in which the vector multiplet scalar $\phi_{9}$ obtains a non-zero VEV, i.e., $\left\langle\phi_{9}\right\rangle \neq 0$. The VEV breaks the $\mathrm{U}(N)$ 
gauge symmetry into its Abelian subgroup $\mathrm{U}(1)^{N}$. Also, it preserves only $\mathrm{SO}(4)_{2}$ of the $\mathrm{SO}(5)_{R}$ symmetry group that we often decompose into $\mathrm{SO}(4)_{2} \simeq \mathrm{SU}(2)_{2 L} \times \mathrm{SU}(2)_{2 R}$.

The $5 \mathrm{~d}$ massive particles preserve the $\mathrm{SO}(4)_{1}$ little group of $\mathrm{SO}(1,4)$, which can be written as $\mathrm{SO}(4)_{1} \simeq \mathrm{SU}(2)_{1 L} \times \mathrm{SU}(2)_{1 R}$. We denote the doublet indices of $\mathrm{SU}(2)_{1 L}$, $\mathrm{SU}(2)_{1 R}, \mathrm{SU}(2)_{2 L}, \mathrm{SU}(2)_{2 R}$ by $\alpha, \dot{\alpha}, a, A$, respectively. The 16 supercharges of $5 \mathrm{~d}$ maximal SYM are then $Q^{\alpha A}, Q^{\dot{\alpha} A}, Q^{\alpha a}, Q^{\dot{\alpha} a}$. There are two types of massive $\frac{1}{2}$-BPS particles in the Coulomb phase: W-bosons and instantons. W-bosons are electrically charged objects under $\mathrm{U}(1)^{N} \subset \mathrm{U}(N)$, preserving $Q^{\dot{\alpha} A}$ and $Q^{\alpha a}$. Their mass is proportional to the Coulomb VEV, i.e., $M=\operatorname{Tr}\left(\left\langle\phi_{9}\right\rangle \cdot \Pi\right)$, where $\Pi$ is the vector of charges with respect to $\mathrm{U}(1)^{N} \subset \mathrm{U}(N)$. Selfdual instantons are solitonic objects having the topological $\mathrm{U}(1)$ charge $k \equiv \frac{1}{8 \pi^{2}} \int_{\mathbf{R}^{4}} \operatorname{Tr}(F \wedge$ $F)$, which is a positive integer. They satisfy the self-duality condition $F_{a b}=\frac{1}{2} \epsilon_{a b c d} F_{c d}$ on the spatial $\mathbf{R}^{4}$ and preserve $Q^{\dot{\alpha} A}$ and $Q^{\dot{\alpha} a}$. The unit instanton mass is $8 \pi^{2} / g_{5}^{2}$ with the gauge coupling $g_{5}$. More generally, W-bosons and instantons can form $\frac{1}{4}$-BPS bound states that preserve $Q^{\dot{\alpha} A}$, with mass

$$
M=\operatorname{Tr}\left(\left\langle\phi_{9}\right\rangle \cdot \Pi\right)+\frac{8 \pi^{2} k}{g_{5}^{2}} .
$$

The line defects can be introduced by adding $N^{\prime} \mathrm{D} 4^{\prime}$-branes which fill the $x^{0}, x^{5}, \cdots, x^{8}$ directions [17]. The open string excitations connecting D4- and D4'-branes induce heavy fermionic probe particles in the $5 \mathrm{~d}$ theory living on the $\mathrm{D} 4$ branes. This will give rise to $\frac{1}{2}$-BPS line defects that will be invariant under the SUSY transformation generated by $Q^{\dot{\alpha} A}$ and $Q^{\alpha a}$. They couple to the 5 d gauge field $\tilde{A}_{\mu}$ and the vector multiplet scalar $\phi_{9}$ through

$$
\mathcal{L}_{1 d}=\int d t \sum_{i=1}^{N^{\prime}} \chi_{i}^{\dagger}\left(\partial_{t}-i \tilde{A}_{t}+\phi_{9}+M_{i}\right) \chi^{i}
$$

where $M_{i}$ is the background gauge field for the $\mathrm{U}(1)^{N^{\prime}} \subset \mathrm{U}\left(N^{\prime}\right)$ flavor symmetry that acts on $\chi_{1}, \cdots, \chi_{N^{\prime}}$. The mass parameter $M_{i}$ of the fermion $\chi_{i}$ sets the energy scale for its excitation. In this paper we will be interested in the partition function of this 5d SYM coupled to the $1 \mathrm{~d}$ line defects, as introduced above. For the case of $N^{\prime}=1$, this was first studied in $[14,15]$. The fugacity variable $e^{-M_{i}}$ counts the number of $\chi_{i}$ excitations. Taking the series expansion in $e^{-M_{i}}$ of the $5 \mathrm{~d} / 1 \mathrm{~d}$ QFT partition function, the individual coefficients correspond to Wilson loop operators in various representations [17, 20,21], as will be explained in section 2.3. In other words, the line defect partition function can be viewed as a generating function of Wilson loops.

\subsection{Review of ADHM quantum mechanics}

The low energy dynamics of $5 \mathrm{~d}$ SYM instantons can be approximated by the $1 \mathrm{~d} \mathcal{N}=(0,4)$ non-linear sigma model whose target space is the instanton moduli space [22]. However, the target space has singularities at which the instanton size shrinks to zero. Since the small instanton configurations are beyond the legitimate regime of $5 \mathrm{~d}$ gauge theory, the UV completion of $5 \mathrm{~d}$ gauge theory prescribes how to resolve the singularities. In particular, the 


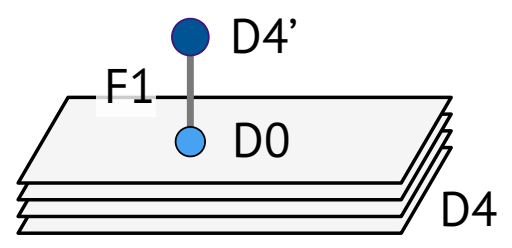

Figure 1. The brane configuration describing $5 \mathrm{~d}$ instantons in the presence of a $5 \mathrm{~d}$ Wilson line defect. See figure 2 for the quiver diagram depicting the D0-brane gauged quantum mechanics.

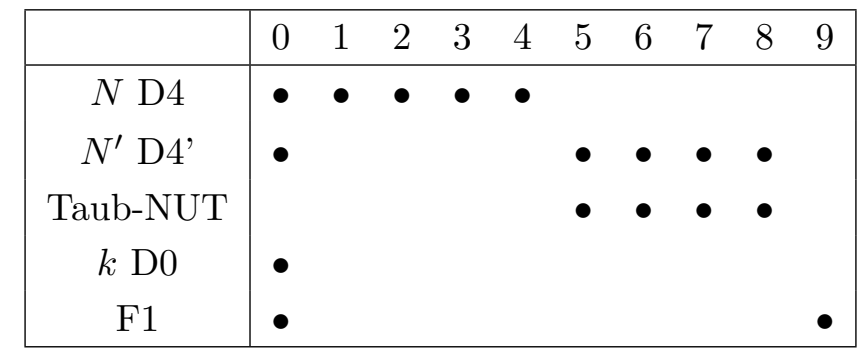

Table 1. Brane configuration for $5 \mathrm{~d} \mathcal{N}=1^{*} \mathrm{U}(N)$ SYM with line defects.

UV completion based on D-brane engineering of the gauge theory motivates the ADHM construction of instantons, realizing the SYM instantons as D0-branes [23].

The dynamics of 5d SYM instantons in the presence of $\frac{1}{2}$-BPS line defect was described in [17]. The insertion of the line defect does not change the static instanton configurations, however, it induces a Lorentz force on slowly moving instantons. The ADHM quantum mechanics of $5 \mathrm{~d}$ instantons in the presence of fermionic line defects is given by the worldvolume gauge theory of D0-branes probing the D4-D4' brane configuration (see figure 1). The spacetime dimensions occupied by this D0-D4-D4' system are as given in table 1, where the Taub-NUT space is introduced along the $x^{5}, \cdots, x^{8}$ directions to realize the mass deformation of $5 \mathrm{~d} \mathcal{N}=1^{*} \mathrm{SYM}$. The UV quantum mechanics corresponding to an instanton configuration with charge $k$ has a $\mathrm{U}(k)$ gauge symmetry. It preserves the $\mathrm{SU}(2)_{1 L} \times \mathrm{SU}(2)_{1 R} \times \mathrm{SU}(2)_{2 L} \times \mathrm{SU}(2)_{2 R}$ global symmetry, where $\mathcal{N}=(0,4)$ R-symmetry group is $\mathrm{SU}(2)_{1 R} \times \mathrm{SU}(2)_{2 R}$. The $\mathrm{U}(N)$ and $\mathrm{U}\left(N^{\prime}\right)$ internal symmetries of D4- and $\mathrm{D} 4{ }^{\prime}-$ branes also appear as the flavor symmetries of the ADHM quantum mechanics.

Various $(0,4)$ multiplets arise from the massless excitations of open strings, contributing to the ADHM quantum mechanics of D0-branes. The D0-D0 strings alone preserve $\mathcal{N}=(8,8)$ supersymmetry and give rise to an $(8,8)$ vector multiplet. One can decompose the $(8,8)$ vector multiplet into the following $(0,4)$ supermultiplets: a vector multiplet $\left(A_{0}, \varphi, \zeta_{\dot{\alpha}}^{A}\right)$, a twisted hypermultiplet $\left(\varphi_{a A}, \chi_{a}^{\dot{\alpha}}\right)$, a hypermultiplet $\left(a_{\alpha \dot{\alpha}}, \lambda_{\alpha}^{A}\right)$, and a Fermi multiplet $\left(\lambda_{\alpha}^{a}\right)$. All of these transform in the adjoint representation of the $\mathrm{U}(k)$ gauge symmetry. The D0-D4 strings provide a $(0,4)$ hypermultiplet $\left(q_{\dot{\alpha}}, \psi^{A}\right)$ and a Fermi multiplet $\left(\psi^{a}\right)$, which combine into a $(4,4)$ hypermultiplet transforming in the bifundamental representation of $\mathrm{U}(k) \times \mathrm{U}(N)$. The above multiplets form the field content of the ADHM quantum mechanics for $5 \mathrm{~d} \mathcal{N}=1^{*} \mathrm{SYM}$ instantons without line defects. With addition of the line defects associated to $N^{\prime} \mathrm{D} 4^{\prime}$-branes, the massless modes of D0-D4 ${ }^{\prime}$ strings further 


\begin{tabular}{|c|c|ccc|}
\hline Type & Fields & $\mathrm{U}(k)$ & $\mathrm{U}(N)$ & $\mathrm{U}\left(N^{\prime}\right)$ \\
\hline vector & $A_{0}, \varphi, \zeta_{\dot{\alpha}}^{A}$ & $\mathbf{a d j}$ & $\mathbf{1}$ & $\mathbf{1}$ \\
twisted & $\varphi_{a A}, \chi_{a}^{\dot{\alpha}}$ & $\mathbf{a d j}$ & $\mathbf{1}$ & $\mathbf{1}$ \\
hyper & $a_{\alpha \dot{\alpha}}, \lambda_{\alpha}^{A}$ & $\mathbf{a d j}$ & $\mathbf{1}$ & $\mathbf{1}$ \\
Fermi & $\lambda_{\alpha}^{a}$ & $\mathbf{a d j}$ & $\mathbf{1}$ & $\mathbf{1}$ \\
hyper & $q_{\dot{\alpha}}, \psi^{A}$ & $\mathbf{k}$ & $\overline{\mathbf{N}}$ & $\mathbf{1}$ \\
Fermi & $\psi^{a}$ & $\mathbf{k}$ & $\overline{\mathbf{N}}$ & $\mathbf{1}$ \\
\hline
\end{tabular}

(a) From the D0-D0 and D0-D4 string modes.

\begin{tabular}{|c|c|ccc|}
\hline Type & Fields & $\mathrm{U}(k)$ & $\mathrm{U}(N)$ & $\mathrm{U}\left(N^{\prime}\right)$ \\
\hline twisted & $\Phi_{A}, \Psi_{\dot{\alpha}}$ & $\mathbf{k}$ & $\mathbf{1}$ & $\overline{\mathbf{N}^{\prime}}$ \\
Fermi & $\Psi_{\alpha}$ & $\mathbf{k}$ & $\mathbf{1}$ & $\overline{\mathbf{N}^{\prime}}$ \\
\hline
\end{tabular}

(b) From the D0-D4' string modes.

\begin{tabular}{|c|c|ccc|}
\hline Type & Fields & $\mathrm{U}(k)$ & $\mathrm{U}(N)$ & $\mathrm{U}\left(N^{\prime}\right)$ \\
\hline Fermi & $\rho$ & $\mathbf{1}$ & $\overline{\mathbf{N}}$ & $\mathbf{N}^{\prime}$ \\
\hline
\end{tabular}

(c) From the D4-D4' string modes.

Table 2. $\mathcal{N}=(0,4)$ multiplets in the ADHM quantum mechanics.

introduce a $(0,4)$ twisted hypermultiplet $\left(\Phi_{A}, \Psi_{\dot{\alpha}}\right)$ and a Fermi multiplet $\left(\Psi_{\alpha}\right)$, transforming in the bifundamental representation of $\mathrm{U}(k) \times \mathrm{U}\left(N^{\prime}\right)$. Finally, the D4-D4' strings also induce non-trivial degrees of freedom to the ADHM quantum mechanics. These correspond to a Fermi multiplet $(\rho)$ transforming in the bifundamental representation of $\mathrm{U}(N) \times \mathrm{U}\left(N^{\prime}\right)$. Although being a singlet of the $\mathrm{U}(k)$ gauge symmetry, it plays a crucial role in writing the SUSY invariant interaction. It is the coupling of this field to the rest, that induces the Lorentz-force on slow moving instantons. The gauged quantum mechanics resulting from the interaction of the above multiplets is shown in table 2 and figure 2.

The Lagrangian of this SUSY quantum mechanics was explicitly constructed in $[17,24]$ by employing $\mathcal{N}=(0,2)$ superfield formalism [25]. For each Fermi multiplet $\eta$, they introduce the potentials $E_{\eta}$ and $J_{\eta}$ as holomorphic functions of $(0,2)$ chiral multiplets in a way that ensures $\mathcal{N}=(0,4)$ enhancement. We decompose the $(0,4)$ vector and (twisted) hypermultiplets into the $(0,2)$ multiplets as follows:

$$
\begin{aligned}
\text { vector }\left(A_{0}, \varphi, \zeta_{\dot{\alpha}}^{A}\right) & \longrightarrow \operatorname{vector} V\left(A_{0}, \varphi, \zeta_{\dot{1}}^{1}\right) \oplus \operatorname{Fermi} \Lambda\left(\zeta_{\dot{2}}^{1}\right) \\
\text { hyper }\left(a_{\alpha \dot{\alpha}}, \lambda_{\alpha}^{A}\right) & \longrightarrow \operatorname{chiral} B\left(a_{11}, \lambda_{1}^{2}\right) \oplus \operatorname{chiral} \tilde{B}^{\dagger}\left(a_{1 \dot{2}}, \lambda_{1}^{1}\right) \\
\text { hyper }\left(q_{\dot{\alpha}}, \psi^{A}\right) & \longrightarrow \operatorname{chiral} q\left(q_{\dot{1}}, \psi^{2}\right) \oplus \operatorname{chiral} \tilde{q}^{\dagger}\left(q_{\dot{2}}, \psi^{1}\right) \\
\text { twisted hyper }\left(\Phi_{A}, \Psi_{\dot{\alpha}}\right) & \longrightarrow \operatorname{chiral} \Phi\left(\Phi_{1}, \Psi_{\dot{1}}\right) \oplus \operatorname{chiral} \tilde{\Phi}^{\dagger}\left(\Phi_{2}, \Psi_{\dot{2}}\right) \\
\text { twisted hyper }\left(\varphi_{a A}, \xi_{a}^{\dot{\alpha}}\right) & \longrightarrow \operatorname{chiral} \varphi\left(\varphi_{11}, \xi_{1}^{\dot{2}}\right) \oplus \operatorname{chiral} \tilde{\varphi}^{\dagger}\left(\varphi_{12}, \xi_{1}^{\dot{1}}\right) .
\end{aligned}
$$

All $(0,4)$ Fermi multiplets are equivalent to $(0,2)$ Fermi multiplets. Following [24], we turn on $J_{\Lambda}\left(E_{\Lambda}\right)$ as the following holomorphic functions of $(0,2)$ chiral multiplets:

$$
J_{\Lambda}=q \tilde{q}+[B, \tilde{B}], \quad E_{\Lambda}=\Phi \tilde{\Phi}+[\varphi, \tilde{\varphi}] .
$$

To meet $\mathcal{N}=(0,2)$ SUSY preserving condition, $\sum_{\eta} \operatorname{Tr}\left(J_{\eta} \cdot E_{\eta}\right)=0$, we also introduce the holomorphic potentials for other Fermi multiplets as follows:

$E_{\psi^{1}}=\tilde{\varphi} q, \quad J_{\psi^{1}}=-\tilde{q} \varphi, \quad E_{\psi^{2}}=\varphi q, \quad J_{\psi^{2}}=\tilde{q} \tilde{\varphi}, \quad E_{\Psi_{1}}=B \Phi, J_{\Psi_{1}}=\tilde{\Phi} \tilde{B}, \quad E_{\Psi_{2}}=-\tilde{B} \Phi, J_{\Psi_{2}}=\tilde{\Phi} B$, $E_{\lambda_{1}^{1}}=[B, \tilde{\varphi}], \quad J_{\lambda_{1}^{1}}=[\tilde{B}, \varphi], \quad E_{\lambda_{1}^{2}}=[B, \varphi], \quad J_{\lambda_{1}^{2}}=-[\tilde{B}, \tilde{\varphi}], \quad E_{\rho}=\tilde{\Phi} q, \quad J_{\rho}=-\tilde{q} \Phi$. 


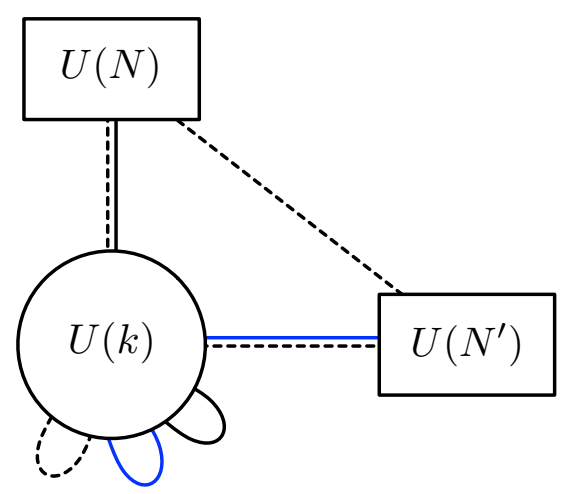

Figure 2. The quiver describing the ADHM quantum mechanics that captures the effect of slow moving $5 \mathrm{~d}$ instantons in the presence of a Wilson line defect. Here solid black lines denote a hypermultiplet, solid blue lines denote a twisted hyper-multiplet, while the dashed lines denote a Fermi multiplet.

Combined with the standard D-term $D=q q^{\dagger}-\tilde{q}^{\dagger} \tilde{q}+\Phi \Phi^{\dagger}-\tilde{\Phi}^{\dagger} \tilde{\Phi}+\left[B, B^{\dagger}\right]-\left[\tilde{B}^{\dagger}, \tilde{B}\right]+$ $\left[\varphi, \varphi^{\dagger}\right]-\left[\tilde{\varphi}^{\dagger}, \tilde{\varphi}\right]$, the bosonic potential $V=\sum_{\eta}\left(\left|E_{\eta}\right|^{2}+\left|J_{\eta}\right|^{2}\right)+\frac{1}{4} D^{2}$ can be arranged into

$$
\begin{aligned}
V= & \frac{1}{4}\left(\left(\sigma^{m}\right)^{\dot{\alpha}}{ }_{\dot{\beta}} q_{\dot{\alpha}} q^{\dagger \dot{\beta}}+\frac{1}{2}\left(\sigma^{m}\right)_{\dot{\beta}}^{\dot{\alpha}}\left[a_{\alpha \dot{\alpha}}, a^{\alpha \dot{\beta}}\right]\right)^{2}+\frac{1}{4}\left(\left(\sigma^{I}\right)^{A}{ }_{B} \Phi_{A} \Phi^{\dagger B}+\frac{1}{2}\left(\sigma^{I}\right)_{B}^{A}\left[\varphi_{a A}, \varphi^{a B}\right]\right)^{2} \\
& +\frac{1}{2}\left|\Phi^{\dagger A} q_{\dot{\alpha}}\right|^{2}+\frac{1}{2}\left|\varphi_{a A} q_{\dot{\alpha}}\right|^{2}+\frac{1}{2}\left|a_{\alpha \dot{\alpha}} \Phi_{A}\right|^{2}+\frac{1}{4}\left|a_{\alpha \dot{\alpha}} \varphi_{a A}-\varphi_{a A} a_{\alpha \dot{\alpha}}\right|^{2}
\end{aligned}
$$

where $m$ and $I$ are triplet indices for $\mathrm{SU}(2)_{1 R}$ and $\mathrm{SU}(2)_{2 R}$, respectively and $\sigma^{m}, \sigma^{I}$ are the corresponding Pauli matrices.

Let us examine the classical moduli space of the ADHM quantum mechanics. The Higgs branch is parameterized by VEVs of the $\mathrm{SU}(2)_{1 R}$ doublet fields, $q_{\dot{\alpha}}$ and $a_{\alpha \dot{\alpha}}$, subject to the following ADHM constraint:

$$
\left(\sigma^{m}\right)_{\dot{\beta}}^{\dot{\alpha}} q_{\dot{\alpha}} q^{\dagger \dot{\beta}}+\frac{1}{2}\left(\sigma^{m}\right)_{\dot{\beta}}^{\dot{\alpha}}\left[a_{\alpha \dot{\alpha}}, a^{\alpha \dot{\beta}}\right]=0 .
$$

All $\Phi_{A}$ and $\varphi_{a A}$ fluctuations become massive, except at small instanton singularities where the VEV $\left\langle q_{\dot{\alpha}}\right\rangle$ is zero. This shows that the Higgs branch corresponds to the $\mathrm{U}(N)$ instanton moduli space. Likewise, the twisted Higgs branch is spanned by VEVs of the $\mathrm{SU}(2)_{2 R}$ doublet fields, $\Phi_{A}$ and $\varphi_{a A}$, which are subject to the following constraint having the same functional form as (2.7):

$$
\left(\sigma^{I}\right)^{A}{ }_{B} \Phi_{A} \Phi^{\dagger B}+\frac{1}{2}\left(\sigma^{I}\right)^{A}{ }_{B}\left[\varphi_{a A}, \varphi^{a B}\right]=0 .
$$

Moving away from singularities $\left\langle\Phi_{A}\right\rangle=0$, the development of the $q_{\dot{\alpha}}$ and $a_{\alpha \dot{\alpha}}$ moduli is also suppressed. So the twisted Higgs branch is the $\mathrm{U}\left(N^{\prime}\right)$ instanton moduli space with exchange of $\mathrm{SO}(4)_{1} \leftrightarrow \mathrm{SO}(4)_{2}$. Finally, the mixed branch emerges when $\left\langle q_{\dot{\alpha}}\right\rangle=0$ and $\left\langle\Phi_{A}\right\rangle=0$, being parameterized by $a_{\alpha \dot{\alpha}}$ and $\varphi_{a A}$. This corresponds to freely moving D0-branes, unbound to any of background D4- or D4'-branes. We remark that only the 
Higgs branch is relevant from the 5d SYM perspective. Other branches emerge due to the extra degrees of freedom introduced for the UV completion of 5d SYM instantons. Their effects on the Witten index must be properly taken into account, to obtain the correct $5 \mathrm{~d}$ SYM observables via the ADHM quantum mechanics. We discuss this issue in detail in section 2.3 .

\subsection{ADHM index with line operators}

We consider the BPS partition function of the $5 \mathrm{~d} / 1 \mathrm{~d}$ coupled system on Omega-deformed $\mathbf{R}^{4} \times S^{1}$, which is given by the following trace formula:

$$
Z_{5 \mathrm{~d} / 1 \mathrm{~d}}=\operatorname{Tr}_{\mathcal{H}_{5 \mathrm{~d}}}\left[(-1)^{F} e^{-\beta H} q^{k} t^{2\left(J_{r}-J_{R}\right)} u^{2 J_{l}} \mu^{2 J_{f}} \prod_{a=1}^{N} w_{a}^{G_{a}} \prod_{i=1}^{N^{\prime}} x_{l}^{Q_{l}}\right] .
$$

It counts the BPS states in $5 \mathrm{~d} \mathcal{N}=1^{*} \mathrm{SYM}$, annihilated by the supercharges $Q^{1 \mathrm{i}}$ and $Q^{2 \dot{2}}$ satisfying $H \sim\left\{Q^{1 \dot{1}}, Q^{2 \dot{2}}\right\}$. Here, $J_{l}, J_{r}, J_{f}, J_{R}$ are the Cartan generators for $\mathrm{SU}(2)_{1 L}, \mathrm{SU}(2)_{1 R}, \mathrm{SU}(2)_{2 L}, \mathrm{SU}(2)_{2 R}$ global symmetries respectively. $G_{a}$ and $Q_{l}$ denote the respective Cartan generators for $5 \mathrm{~d} \mathrm{U}(N)$ gauge and $\mathrm{U}\left(N^{\prime}\right)$ flavor symmetry. $k$ is the topological U(1) charge for 5d SYM instantons which is conjugate to the instanton fugacity $q \equiv \exp \left(-8 \pi^{2} / g_{5 \mathrm{~d}}^{2}\right)$. For all combinations of the Cartan generators commuting with $Q^{1 \dot{1}}$ and $Q^{2 \dot{2}}$, the conjugate fugacity variables are introduced as follows:

$$
t=e^{-\epsilon_{+}}, \quad u=e^{-\epsilon_{-}}, \quad \mu=e^{-m}, \quad x_{l}=e^{-M_{l}}, \quad w_{a}=e^{-\alpha_{a}} .
$$

$\epsilon_{+} \equiv \frac{\epsilon_{1}+\epsilon_{2}}{2}$ and $\epsilon_{-} \equiv \frac{\epsilon_{1}-\epsilon_{2}}{2}$ introduce the Omega-deformation of the spatial $\mathbf{R}^{4}$ in the $5 \mathrm{~d}$ gauge theory. $m$ is the mass of the $\mathcal{N}=1$ adjoint hypermultiplet in the $\mathcal{N}=2$ vector multiplet, realizing the mass deformation of $\mathcal{N}=1^{*}$ SYM. $\alpha_{a}$ parametrizes the VEV of the $\mathcal{N}=1$ vector multiplet scalar, i.e., $\left\langle\phi_{9}\right\rangle=\operatorname{diag}\left(\alpha_{1}, \alpha_{2}, \cdots, \alpha_{N}\right)$, that breaks $\mathrm{U}(N)$ into $\mathrm{U}(1)^{N}$. These chemical potentials deform the $5 \mathrm{~d} / 1 \mathrm{~d}$ coupled system by acting as infrared regulators which generate a mass gap for the instanton zero modes $[26,27] . M_{l}$ is the mass parameter for the $1 \mathrm{~d}$ defect fermion, breaking the $\mathrm{U}\left(N^{\prime}\right)$ flavor symmetry into its maximal torus.

The partition function in (2.9) represents the grand canonical ensemble of all multiinstanton BPS states. One can write the full partition function as the sum over all instanton sectors, multiplied by the perturbative partition function $Z_{\text {pert }}$ that captures the bound states of W-bosons. It implies that we have to sum over the full tower of D0-branes, thereby giving us

$Z_{\mathrm{ADHM}}^{\left(N, N^{\prime}\right)} \equiv \sum_{k=0}^{\infty} q^{k} Z_{k}^{\left(N, N^{\prime}\right)} \quad$ with $Z_{k}^{\left(N, N^{\prime}\right)} \equiv \operatorname{Tr}_{\mathcal{H}_{1 \mathrm{~d}}^{(k)}}\left[(-1)^{F} e^{-\beta H} t^{2\left(J_{r}-J_{R}\right)} u^{2 J_{l}} \mu^{2 J_{f}} \prod_{a=1}^{N} w_{a}^{G_{a}} \prod_{i=1}^{N^{\prime}} x_{l}^{Q_{l}}\right]$

where the trace is taken over all the BPS states in the ADHM quantum mechanics of $k$ D0-branes. The corresponding Witten index $Z_{k}^{\left(N, N^{\prime}\right)}$ can be computed by using the results of $[28-30]$. 
Let us evaluate the gauge theory path integral in the weak coupling regime $g_{1} \rightarrow 0$ of the UV quantum mechanics, reducing it down to Gaussian integrals around saddle points. The saddle points are parameterized by the complexified holonomy $\phi=i \beta A_{0}+\beta \varphi$, made of the gauge field $A_{0}$ and the scalar $\varphi$ in the vector multiplet. We denote the eigenvalues of $\phi$ by $\phi_{1}, \cdots, \phi_{k}$, each of which takes value on a cylinder of radius $\frac{\beta}{2 \pi}$. Performing the Gaussian integrals over massive fluctuations we remain with the zero mode integration of the resulting 1-loop determinant:

$$
Z_{k}^{\left(N, N^{\prime}\right)}=\frac{1}{k !} \oint\left[\prod_{i=1}^{k} \frac{d \phi_{i}}{2 \pi i}\right] Z_{k}^{\mathrm{vec}}\left(\phi, \alpha, \epsilon_{1,2}\right) Z_{k}^{\mathrm{adj}}\left(\phi, \alpha, m, \epsilon_{1,2}\right) \prod_{l=1}^{N^{\prime}} Z_{k}^{1 \mathrm{~d}}\left(\phi, \alpha, M_{l}, \epsilon_{1,2}\right),
$$

where (by using $\operatorname{sh}(x) \equiv 2 \sinh (x / 2))$

$$
\begin{aligned}
Z_{k}^{\mathrm{vec}}\left(\phi, \alpha, \epsilon_{1,2}\right) & =\prod_{\substack{j \neq i \\
i, j=1}}^{k} \operatorname{sh}\left(\phi_{i j}\right) \prod_{i, j=1}^{k} \frac{\operatorname{sh}\left(2 \epsilon_{+}+\phi_{i j}\right)}{\operatorname{sh}\left(\epsilon_{+} \pm \epsilon_{-}+\phi_{i j}\right)} \prod_{i=1}^{k} \prod_{a=1}^{N} \frac{1}{\operatorname{sh}\left(\epsilon_{+} \pm\left(\phi_{i}-\alpha_{a}\right)\right)}, \\
Z_{k}^{\mathrm{adj}}\left(\phi, \alpha, m, \epsilon_{1,2}\right) & =\prod_{i, j=1}^{k} \frac{\operatorname{sh}\left(m \pm \epsilon_{-}+\phi_{i j}\right)}{\operatorname{sh}\left(-\epsilon_{+} \pm m+\phi_{i j}\right)} \prod_{i=1}^{k} \prod_{a=1}^{N} \operatorname{sh}\left(m \pm\left(\phi_{i}-\alpha_{a}\right)\right) \\
Z_{k}^{1 \mathrm{~d}}\left(\phi, \alpha, M_{l}, \epsilon_{1,2}\right) & =\prod_{i=1}^{k} \frac{\operatorname{sh}\left(\epsilon_{-} \pm\left(\phi_{i}-M_{l}\right)\right)}{\operatorname{sh}\left(-\epsilon_{+} \pm\left(\phi_{i}-M_{l}\right)\right)} \prod_{a=1}^{N} \operatorname{sh}\left(\alpha_{a}-M_{l}\right) .
\end{aligned}
$$

The zero mode integration translates into a contour integral over the space of $\phi$ eigenvalues. The proper choice of the integral contour is done by the Jeffrey-Kirwan residue operation [28-30]. It refers to an auxiliary $k$-dimensional vector $\eta$, although the final residue sum becomes independent of $\eta$. We choose $\eta=(1, \cdots, 1)$ to find an explicit expression of the residues.

For the special case of $N^{\prime}=0$, the Jeffrey-Kirwan residues are labeled by $N$-tuples of Young tableaux $\left(Y_{1}, \cdots, Y_{N}\right)$ such that $\sum_{a=1}^{N}\left|Y_{a}\right|=k$ [26-28]. We uniquely assign the numbers $1,2, \cdots, k$ to each of the boxes. Suppose the $i$ 'th box is at the $m$ 'th row and $n$ 'th column of $Y_{a}$. This represents the following pole location for an integral variable $\phi_{i}$ :

$$
\phi_{i}=\alpha_{a}-\epsilon_{+}-(n-1) \epsilon_{1}-(m-1) \epsilon_{2} .
$$

The sum over all the Jeffrey-Kirwan residues can be written as [31, 32]

$$
Z_{k}^{(N, 0)}=\sum_{\sum_{a}\left|Y_{a}\right|=k} \prod_{a, b=1}^{N} \prod_{s \in Y_{a}} \frac{\operatorname{sh}\left(E_{a b}(s)+m-\epsilon_{+}\right) \operatorname{sh}\left(E_{a b}(s)-m-\epsilon_{+}\right)}{\operatorname{sh}\left(E_{a b}(s)\right) \operatorname{sh}\left(E_{a b}(s)-2 \epsilon_{+}\right)}
$$

where $E_{a b}(s) \equiv \alpha_{a}-\alpha_{b}-\epsilon_{1} h_{a}(s)+\epsilon_{2}\left(v_{b}(s)+1\right) . v_{b}(s)$ is the vertical distance from $s$ to the bottom of $Y_{b}$. $h_{a}(s)$ denotes the horizontal distance from $s$ to the right end of $Y_{a}$. (2.15) is the $k$-instanton correction to the partition function of $5 \mathrm{~d} \mathcal{N}=1^{*} \mathrm{U}(N) \mathrm{SYM}$ without defects.

Similarly, for another special case of $N=0$, the Jeffrey-Kirwan residues are classified by $N^{\prime}$-tuples of Young tableaux $\left(Y_{1}, \cdots, Y_{N^{\prime}}\right)$ with $\sum_{a=1}^{N^{\prime}}\left|Y_{a}\right|=k$ [26-28]. The pole location 
and residue sum are identical to (2.14) and (2.15), except for the role reversal specified by $\mathrm{U}(N) \leftrightarrow \mathrm{U}\left(N^{\prime}\right)$ and $\mathrm{SO}(4)_{1} \leftrightarrow \mathrm{SO}(4)_{2}$, i.e.,

$$
\left(N,\left\{\alpha_{a}\right\}, \epsilon_{+}, \epsilon_{-}, m\right) \leftrightarrow\left(N^{\prime},\left\{M_{l}\right\},-\epsilon_{+}, m, \epsilon_{-}\right) .
$$

At this point we also note that in the $N=0$ limit, we would have expected the ADHM quantum mechanics to become trivial, i.e. $Z_{5 \mathrm{~d} / 1 \mathrm{~d}}=1$. However it turns out that this is not the case for the partition function, $Z_{\mathrm{ADHM}}^{\left(0, N^{\prime}\right)}$, in the current set-up. This is because when $N=0, Z_{k}^{\left(0, N^{\prime}\right)}$ captures the bound states of $k$ D0-branes with the $N^{\prime}$ D4'-branes. In general, the multi-particle index of the ADHM quantum mechanics captures not only the BPS states of the $5 \mathrm{~d} / 1 \mathrm{~d}$ coupled system, but also the extra bound states of D0-branes decoupled from the QFT dynamics on D4-branes. In order to identify the correct QFT observable in section 2.3, we need to remove the extra BPS states that arise as a result of the UV completion of the non-linear sigma model onto the instanton moduli space.

We now turn to the general case of $N, N^{\prime} \neq 0$. All the non-vanishing Jeffrey-Kirwan residues are classified by $\left(N+N^{\prime}\right)$-tuples of Young tableaux $\vec{Y}=\left(Y_{1}, \cdots, Y_{N} \mid Y_{N+1}, \cdots\right.$, $\left.Y_{N+N^{\prime}}\right)$ satisfying $\sum_{a=1}^{N+N^{\prime}}\left|Y_{a}\right|=k$. We label all the $k$ boxes by the uniquely assigned integers $1, \cdots, k$. Suppose the $i$ 'th and $j$ 'th boxes are located at the $m^{\prime}$ th row and $n^{\prime}$ th column of $Y_{a \leq N}$ and $Y_{l>N}$, respectively. These boxes encode the following information on the pole locations for integral variables $\phi_{i}$ and $\phi_{j}$ :

$$
\phi_{i}=\alpha_{a}-\epsilon_{+}-(n-1) \epsilon_{1}-(m-1) \epsilon_{2}, \quad \phi_{j}=M_{l}+\epsilon_{+}-(n-1) \epsilon_{3}-(m-1) \epsilon_{4},
$$

with $\epsilon_{3} \equiv-\epsilon_{+}+m$ and $\epsilon_{4} \equiv-\epsilon_{+}-m$. No poles can be further developed at other locations, such as $\alpha_{a}-\epsilon_{+}-(n-1) \epsilon_{1}-(m-1) \epsilon_{2}-\epsilon_{3,4}$ or $M_{l}+\epsilon_{+}-(n-1) \epsilon_{3}-(m-1) \epsilon_{4}-\epsilon_{1,2}$ due to the suppressing factors

$$
\prod_{i=1}^{k}\left(\prod_{a=1}^{N} \operatorname{sh}\left(m \pm\left(\phi_{i}-\alpha_{a}\right)\right) \prod_{l=1}^{N^{\prime}} \operatorname{sh}\left(+\epsilon_{-} \pm\left(\phi_{i}-M_{l}\right)\right) \prod_{j=1}^{k} \operatorname{sh}\left(m \pm \epsilon_{-}+\phi_{i j}\right)\right)
$$

in the numerator of (2.12). The JK residue sum can be written as the following expression:

$$
\begin{aligned}
Z_{k}^{\left(N, N^{\prime}\right)}= & \sum_{\vec{Y}}\left[\prod_{a, b=1}^{N} \prod_{s \in Y_{a}}\left(\frac{\operatorname{sh}\left(E_{a b}(s)+m-\epsilon_{+}\right) \operatorname{sh}\left(E_{a b}(s)-m-\epsilon_{+}\right)}{\operatorname{sh}\left(E_{a b}(s)\right) \operatorname{sh}\left(E_{a b}(s)-2 \epsilon_{+}\right)} \prod_{p=1}^{N^{\prime}} \frac{\operatorname{sh}\left(+\epsilon_{-} \pm\left(\phi(s)-M_{p}\right)\right)}{\operatorname{sh}\left(-\epsilon_{+} \pm\left(\phi(s)-M_{p}\right)\right)}\right)\right. \\
& \times \prod_{p, q=1}^{N^{\prime}} \prod_{s \in Y_{p+N}}\left(\frac{\operatorname{sh}\left(F_{p q}(s)+\epsilon_{-}+\epsilon_{+}\right) \operatorname{sh}\left(F_{p q}(s)-\epsilon_{-}+\epsilon_{+}\right)}{\operatorname{sh}\left(F_{p q}(s)\right) \operatorname{sh}\left(F_{p q}(s)+2 \epsilon_{+}\right)} \prod_{a=1}^{N} \frac{\operatorname{sh}\left(m \pm\left(\phi(s)-\alpha_{a}\right)\right)}{\operatorname{sh}\left(\epsilon_{+} \pm\left(\phi(s)-\alpha_{a}\right)\right)}\right) \times \prod_{a=1}^{N} \prod_{p=1}^{N^{\prime}} \operatorname{sh}\left(\alpha_{a}-M_{p}\right) \\
& \left.\times \prod_{a=1}^{N} \prod_{p=1}^{N^{\prime}}\left(\prod_{\substack{s_{1} \in Y_{a} \\
s_{2} \in Y_{p+N}}} \frac{\operatorname{sh}\left( \pm\left(\phi\left(s_{1}\right)-\phi\left(s_{2}\right)\right)\right) \operatorname{sh}\left(2 \epsilon_{+} \pm\left(\phi\left(s_{1}\right)-\phi\left(s_{2}\right)\right)\right) \operatorname{sh}\left(m \pm \epsilon_{-} \pm\left(\phi\left(s_{1}\right)-\phi\left(s_{2}\right)\right)\right)}{\operatorname{sh}\left(\epsilon_{+} \pm \epsilon_{-} \pm\left(\phi\left(s_{1}\right)-\phi\left(s_{2}\right)\right)\right) \operatorname{sh}\left(-\epsilon_{+} \pm m \pm\left(\phi\left(s_{1}\right)-\phi\left(s_{2}\right)\right)\right)}\right)\right],
\end{aligned}
$$

where $F_{p q}(s) \equiv M_{p}-M_{q}-\epsilon_{3} h_{p}(s)+\epsilon_{4}\left(v_{q}(s)+1\right) . \phi(s)$ denotes the pole location $(2.17)$ for the box $s$. We remark that the contribution from the $k=0$ sector is not 1 but rather $\prod_{a=1}^{N} \prod_{p=1}^{N^{\prime}} \operatorname{sh}\left(\alpha_{a}-M_{p}\right)$, which captures the fermionic excitation of the D4-D4' strings. 
Before we conclude the section, let us mention that the partition function $Z_{\mathrm{ADHM}}^{\left(N, N^{\prime}\right)}$ of the D0-D4-D4' system can also be interpreted as the $N^{\prime}$-th qq-character $X_{N^{\prime}}^{\mathrm{U}(N)}$ of the five-dimensional $\mathcal{N}=1^{*} \mathrm{U}(N)$ theory introduced in [14] (as already noticed in [15] for the $N^{\prime}=1$ case):

$$
Z_{\mathrm{ADHM}}^{\left(N, N^{\prime}\right)}=X_{N^{\prime}}^{\mathrm{U}(N)}\left(M_{1}, \ldots, M_{N^{\prime}}\right),
$$

where we made the dependence on the $M_{l}$ parameters explicit as customary when dealing with qq-characters. Of particular importance is the $N^{\prime}=1$ (aka fundamental) qq-character $X_{1}^{\mathrm{U}(N)}\left(M_{1}\right)$, which can be used to derive Dyson-Schwinger equations for the five-dimensional theory, while the role of $N^{\prime}>1$ (aka higher) qq-characters is less understood: our analysis in section 2.3 will hopefully clarify their meaning a little, by showing that all qq-characters can be interpreted in terms of Wilson loops in arbitrary representations. An important property of the qq-characters is that they can be written as rational functions of another set of observables for the $5 \mathrm{~d} \mathcal{N}=1^{*} \mathrm{U}(N)$ theory known as $y$-observables: these can be thought as the instanton-corrected vacuum expectation values of $y\left(M_{l}\right) \sim \operatorname{det}\left(e^{M_{l}}-e^{\phi}\right)$, with $\phi$ being the combination of the real vector multiplet scalar and the time component of the gauge field. From the ADHM quantum mechanics point of view, the correlator of $n$ such observables (or their inverse) at positions $x_{j}$ can be computed as [15]

$$
\prod_{j=1}^{n} y^{s_{j}}\left(x_{j}\right)=\sum_{k \geqslant 0} q^{k} \frac{1}{k !} \oint\left[\prod_{i=1}^{k} \frac{d \phi_{i}}{2 \pi i}\right] Z_{k}^{\mathrm{vec}} Z_{k}^{\mathrm{adj}} \prod_{j=1}^{n}\left[Z_{k}^{1 \mathrm{~d}}\left(x_{j}\right)\right]^{s_{j}}, \quad s_{j}= \pm 1,
$$

where in the integrand we have the functions defined in (2.13) (although we suppressed the dependence on $\alpha_{a}, \epsilon_{ \pm}, m$ for brevity). The contour integral is again evaluated by summing over residues at those poles which are labeled by $N$-tuples of the Young tableaux (2.14): this pole prescription then distinguishes the 1-point correlator $y\left(M_{1}\right)$ from the fundamental qq-character $X_{1}^{\mathrm{U}(N)}\left(M_{1}\right)$, which is defined by the same contour integral formula but receives contributions from a larger set of poles (2.17). In more detail, in terms of $y$-observables the fundamental qq-character reads [14]

$$
\begin{aligned}
x_{1}^{\mathrm{U}(N)}\left(M_{1}\right)= & y\left(M_{1}\right) \sum_{\lambda} q^{|\lambda|} \prod_{s \in \lambda} S\left(\left(m-\epsilon_{+}\right)(h(s)+1)+\left(m+\epsilon_{+}\right) v(s)\right) \\
& \prod_{s \in \lambda} \frac{y\left(M_{1}-m-\epsilon_{+}+\sigma(s)\right) y\left(M_{1}+m-\epsilon_{+}+\sigma(s)\right)}{y\left(M_{1}+\sigma(s)\right) y\left(M_{1}-2 \epsilon_{+}+\sigma(s)\right)} \\
= & y\left(M_{1}\right)+q S\left(m-\epsilon_{+}\right) \frac{y\left(M_{1}-m-\epsilon_{+}\right) y\left(M_{1}+m-\epsilon_{+}\right)}{y\left(M_{1}-2 \epsilon_{+}\right)}+\ldots ;
\end{aligned}
$$

here $\lambda$ is a Young tableau with any number of boxes, $h(s)$ and $v(s)$ are the distances from the position $(i, j)$ of the box $s \in \lambda$ to the right and bottom ends of the tableau $\lambda$ respectively, and

$$
\sigma(s)=i\left(m-\epsilon_{+}\right)-j\left(m+\epsilon_{+}\right)+2 \epsilon_{+}, \quad S(x)=\frac{\operatorname{sh}\left(x+\epsilon_{1}\right) \operatorname{sh}\left(x+\epsilon_{2}\right)}{\operatorname{sh}(x) \operatorname{sh}\left(x+2 \epsilon_{+}\right)} .
$$


Higher qq-characters can be expressed as rational functions of $y$-observables in a similar way:

$$
\begin{aligned}
& X_{N^{\prime}}^{\mathrm{U}(N)}\left(M_{1}, \ldots, M_{N^{\prime}}\right)= \\
& \quad \prod_{p=1}^{N^{\prime}} y\left(M_{p}\right) \sum_{\vec{\lambda}} q^{\sum_{p=1}^{N^{\prime}}\left|\lambda_{p}\right|} \prod_{p, q=1}^{N^{\prime}} \prod_{s \in \lambda_{p}} S\left(F_{p q}(s)\right) \prod_{p=1}^{N^{\prime}} \prod_{s \in \lambda_{p}} \frac{y\left(M_{p}-m-\epsilon_{+}+\sigma(s)\right) y\left(M_{p}+m-\epsilon_{+}+\sigma(s)\right)}{y\left(M_{p}+\sigma(s)\right) y\left(M_{p}-2 \epsilon_{+}+\sigma(s)\right)},
\end{aligned}
$$

with $\vec{\lambda}=\left(\lambda_{1}, \ldots, \lambda_{N^{\prime}}\right)$ being an $N^{\prime}$-tuple of Young tableaux and

$$
F_{p q}(s)=M_{p}-M_{q}+\left(m-\epsilon_{+}\right)\left(h_{p}(s)+1\right)+\left(m+\epsilon_{+}\right) v_{q}(s) .
$$

\subsection{The Wilson loop observable}

Recall that the multi-particle partition function $Z_{\mathrm{ADHM}}^{\left(N, N^{\prime}\right)}$ of D0-branes not only captures the BPS states in the $5 \mathrm{~d} / 1 \mathrm{~d}$ coupled system, but also receives extra contribution depending on the UV completion of the QFT. Since the ADHM prescription is equivalent to the string theory embedding of the $5 \mathrm{~d} / 1 \mathrm{~d}$ QFT, i.e., the D0-D4-D4' brane system, we expect the extra contribution is associated to the bound states of D0- and D4'-branes. This is obvious for the simple case of $N=0$, where we get (2.15) after (2.16). Now we consider $Z_{\mathrm{ADHM}}$ for a general case and construct the QFT observable out of it.

The multi-particle partition function $Z_{\mathrm{ADHM}}^{\left(N, N^{\prime}\right)}$ starts with $\prod_{a=1}^{N} \prod_{l=1}^{N^{\prime}} \operatorname{sh}\left(\alpha_{a}-M_{l}\right)$ as the $q^{0}$ correction. Imposing the $\mathrm{SU}(N)$ and $\mathrm{SU}\left(N^{\prime}\right)$ traceless conditions $\sum_{a=1}^{N} \alpha_{a}=\sum_{l=1}^{N^{\prime}} M_{l}=$ 0 , it decomposes into the following sum over some irreducible representations $\left(\mathbf{R}, \mathbf{R}^{\prime}\right)$ of $\mathrm{SU}(N) \times \mathrm{SU}\left(N^{\prime}\right):$

$$
Z_{0}^{\left(N, N^{\prime}\right)}=\prod_{a=1}^{N} \prod_{l=1}^{N^{\prime}} \operatorname{sh}\left(\alpha_{a}-M_{l}\right)=\sum_{\left(\mathbf{R}, \mathbf{R}^{\prime}\right)} n_{\mathbf{R}, \mathbf{R}^{\prime}} \cdot \chi_{\mathbf{R}}^{\mathrm{SU}(N)}\left(\alpha_{a}\right) \chi_{\mathbf{R}^{\prime}}^{\mathrm{SU}\left(N^{\prime}\right)}\left(M_{l}\right)
$$

where $\chi_{\mathbf{R}}^{G}$ denotes the character of the Lie group $G$ in the representation $\mathbf{R}$. Also, $n_{\mathbf{R}, \mathbf{R}^{\prime}}$ is an integer counting the degeneracy of the representations. This is also the $5 \mathrm{~d} / 1 \mathrm{~d}$ QFT partition function in (2.9) at zero instanton order, as the issue of extra bound states does not exist. Henceforth, unless explicitly mentioned, we will always impose the $\mathrm{SU}(N)$ and $\mathrm{SU}\left(N^{\prime}\right)$ traceless conditions $\sum_{a=1}^{N} \alpha_{a}=\sum_{l=1}^{N^{\prime}} M_{l}=0$.

At higher $q$, we expect that, just like the right most expression in (2.25), each summand in $Z_{\mathrm{ADHM}}^{\left(N, N^{\prime}\right)}$ will correspond to a product of individual Wilson loops in appropriate representations of $\mathrm{SU}(N)$ and $\mathrm{SU}\left(N^{\prime}\right)$. The only difference will be that the $\mathrm{SU}(N), \mathrm{SU}\left(N^{\prime}\right)$ characters $\chi_{\mathbf{R}}^{\mathrm{SU}(N)}\left(\alpha_{a}\right), \chi_{\mathbf{R}^{\prime}}^{\mathrm{SU}\left(N^{\prime}\right)}\left(M_{l}\right)$ will now incur instanton corrections to become $\mathcal{W}_{\mathbf{R}}^{\mathrm{U}(N)}, \mathcal{W}_{\mathbf{R}^{\prime}}^{\mathrm{U}\left(N^{\prime}\right)}$ respectively: ${ }^{1}$

$$
Z_{\mathrm{ADHM}}^{\left(N, N^{\prime}\right)}=\sum_{\left(\mathbf{R}, \mathbf{R}^{\prime}\right)} n_{\mathbf{R}, \mathbf{R}^{\prime}} \cdot \mathcal{W}_{\mathbf{R}}^{\mathrm{U}(N)} \cdot \mathcal{W}_{\mathbf{R}^{\prime}}^{\mathrm{U}\left(N^{\prime}\right)} .
$$

\footnotetext{
${ }^{1}$ The reason to switch from $\mathrm{SU}(N)$ in the superscript of $\chi_{\mathbf{R}}^{\mathrm{SU}(N)}$ to $\mathrm{U}(N)$ in the superscript of $\mathcal{W}_{\mathbf{R}}^{\mathrm{U}(N)}$ is because even after imposing the $\mathrm{SU}(N)$ traceless condition by hand, the $q$ corrected version of $\chi_{\mathbf{R}}^{\mathrm{SU}(N)}$ contains a U(1) factor (given explicitly in (2.27)) which needs to be appropriately handled.
} 
We believe that $\mathcal{W}_{\mathbf{R}}^{\mathrm{U}(N)}$ captures corrections from the D0-D4 bound states while the $q$ corrections in $\mathcal{W}_{\mathbf{R}^{\prime}}^{\mathrm{U}\left(N^{\prime}\right)}$ come from the D0-D $4^{\prime}$ bound states. This is motivated from the moduli space analysis of section 2.1, where we show that, in the IR, the Higgs branch and the twisted Higgs branch form individual quantum systems decoupled from each other.

The QFT observable $\mathcal{W}_{\mathbf{R}}^{\mathrm{U}(N)}$ is the Wilson line of $5 \mathrm{~d} \mathrm{U}(N)$ SYM engineered from $N$ D4-branes, where the effect of the overall $\mathrm{U}(1)$ is just to include the $\mathrm{U}(1)$ instanton partition function $[33-35]:^{2}$

$$
\mathcal{W}^{\mathrm{U}(1)} \equiv \mathrm{PE}\left[\frac{t\left(-u-u^{-1}+\mu+\mu^{-1}\right)}{(1-t u)\left(1-t u^{-1}\right)} \frac{q}{1-q}\right]
$$

Similarly, $\mathcal{W}_{\mathbf{R}^{\prime}}^{\mathrm{U}\left(N^{\prime}\right)}$ can be interpreted as the Wilson line of $5 \mathrm{~d} \mathrm{SU}\left(N^{\prime}\right) \mathrm{SYM}$ engineered from $N^{\prime} \mathrm{D} 4^{\prime}$-branes, multiplied by the U(1) factor $\left.\mathcal{W}^{\mathrm{U}\left(1^{\prime}\right)} \equiv \mathcal{W}^{\mathrm{U}(1)}\right|_{t \rightarrow t^{-1}, u \leftrightarrow \mu}$ for the D4'-branes. If $N=N^{\prime}$ and $\mathbf{R}=\mathbf{R}^{\prime}$, the observables $\mathcal{W}_{\mathbf{R}}^{\mathrm{U}(N)}$ and $\mathcal{W}_{\mathbf{R}^{\prime}}^{\mathrm{U}\left(N^{\prime}\right)}$ have the same functional form up to the fugacity exchange (2.16). Once we have decomposed $Z_{\mathrm{ADHM}}^{\left(N, N^{\prime}\right)}$ according to (2.26), the $5 \mathrm{~d} / 1 \mathrm{~d}$ QFT partition function (2.9) can be obtained by suppressing the $q$ correction in $\mathcal{W}_{\mathbf{R}^{\prime}}^{\mathrm{U}\left(N^{\prime}\right)}$ as follows:

$$
Z_{5 \mathrm{~d} / 1 \mathrm{~d}}=\sum_{\left(\mathbf{R}, \mathbf{R}^{\prime}\right)} n_{\mathbf{R}, \mathbf{R}^{\prime}} \cdot \mathcal{W}_{\mathbf{R}}^{\mathrm{U}(N)} \cdot\left(\left.\mathcal{W}_{\mathbf{R}^{\prime}}^{\mathrm{U}\left(N^{\prime}\right)}\right|_{q \rightarrow 0}\right)=\sum_{\left(\mathbf{R}, \mathbf{R}^{\prime}\right)} n_{\mathbf{R}, \mathbf{R}^{\prime}} \cdot \mathcal{W}_{\mathbf{R}}^{\mathrm{U}(N)} \cdot \chi_{\mathbf{R}^{\prime}\left(N^{\prime}\right)}^{\mathrm{SU}} .
$$

Let us specialize to the particular case of $N^{\prime}=1$ which was first studied in [15]. It is convenient to keep the $\mathrm{U}(1)$ flavor fugacity alive, such that $x_{1} \neq 1$. The multi-particle index $Z_{\mathrm{ADHM}}^{(N, 1)}$ is the generating function of the minuscule Wilson lines with the overall dressing factor $\mathcal{W}^{\mathrm{U}\left(1^{\prime}\right)}$, i.e.,

$$
Z_{\mathrm{ADHM}}^{(N, 1)}=(-1)^{N} x_{1}^{-N / 2} \sum_{i=0}^{N}\left(-x_{1}\right)^{i} \cdot \mathcal{W}_{\wedge^{i}}^{\mathrm{U}(N)} \mathcal{W}^{\mathrm{U}\left(1^{\prime}\right)},
$$

where the subscript $\bigwedge^{n}$ denotes the rank- $n$ antisymmetric representation. We identified all the $\mathrm{SU}(N)$ Wilson loops with $N \leq 4$ up to 2 instanton corrections. However, for the sake of brevity, we will only display results up to $q^{1}$ order. Furthermore, we will normalize the observable by the partition function of $\mathrm{U}(N)$ SYM which we denote by $\mathcal{W}_{\mathbf{1}}^{\mathrm{U}(N)}$. All results are expressed in terms of the irreducible characters $\chi_{\mathbf{R}}^{l}, \chi_{\mathbf{R}}^{f}, \chi_{\mathbf{R}}^{r}, \chi_{\mathbf{R}}^{\mathrm{SU}(N)}$ of the $\mathrm{SU}(2)_{1 L}, \mathrm{SU}(2)_{2 L}, \mathrm{SU}(2)_{r} \subset \mathrm{SU}(2)_{1 R} \times \mathrm{SU}(2)_{2 R}, \mathrm{SU}(N)$ symmetries.

$$
\begin{aligned}
\frac{\mathcal{W}_{\mathbf{2}}^{\mathrm{U}(2)}}{\mathcal{W}_{\mathbf{1}}^{\mathrm{U}(2)}}= & \chi_{\mathbf{2}}^{\mathrm{SU}(2)}-q \cdot \frac{t^{2} \cdot\left(\chi_{\mathbf{2}}^{f}-\chi_{\mathbf{2}}^{l}\right)\left(\chi_{\mathbf{2}}^{f}-\chi_{\mathbf{2}}^{r}\right)}{\left(1-t^{2} w_{1}^{ \pm 2}\right)} \cdot \chi_{\mathbf{2}}^{\mathrm{SU}(2)}+\mathcal{O}\left(q^{2}\right) \\
\frac{\mathcal{W}_{\mathbf{3}}^{\mathrm{U}(3)}}{\mathcal{W}_{\mathbf{1}}^{\mathrm{U}(3)}}= & \chi_{\mathbf{3}}^{\mathrm{SU}(3)}-q \cdot \frac{t^{6} \cdot\left(\chi_{\mathbf{2}}^{f}-\chi_{\mathbf{2}}^{l}\right)\left(\chi_{\mathbf{2}}^{f}-\chi_{\mathbf{2}}^{r}\right)}{\prod_{i \neq j}\left(1-t^{2} w_{i} w_{j}^{-1}\right)}\left(\chi_{\mathbf{2} \mathbf{4}}^{\mathrm{SU}(3)}+\left(2-\chi_{\mathbf{3}}^{r}\right) \chi_{\mathbf{1 5}}^{\mathrm{SU}(3)}-\left(\chi_{\mathbf{2}}^{f} \chi_{\mathbf{2}}^{r}+2 \chi_{\mathbf{3}}^{r}-1\right) \chi_{\overline{\mathbf{6}}}^{\mathrm{SU}(3)}\right. \\
& \left.+\left(\chi_{\mathbf{2}}^{f} \chi_{\mathbf{4}}^{r}+\chi_{\mathbf{5}}^{r}+2\right) \chi_{\mathbf{3}}^{\mathrm{SU}(3)}\right)+\mathcal{O}\left(q^{2}\right)
\end{aligned}
$$

\footnotetext{
${ }^{2}$ The Plethystic exponential is defined as $\mathrm{PE}\left[f\left(q, t, u, \mu, w_{a}, x_{l}\right)\right] \equiv \exp \left(\sum_{n=1}^{\infty} \frac{1}{n} f\left(q^{n}, t^{n}, u^{n}, \mu^{n}, w_{a}^{n}, x_{l}^{n}\right)\right)$.
} 


$$
\begin{aligned}
& \frac{\mathcal{W}_{\mathbf{4}}^{\mathrm{U}(4)}}{\mathcal{W}_{\mathbf{1}}^{\mathrm{U}(4)}}=\chi_{\mathbf{4}}^{\mathrm{SU}(4)}+q \cdot \frac{t^{12} \cdot\left(\chi_{\mathbf{2}}^{f}-\chi_{\mathbf{2}}^{l}\right)\left(\chi_{\mathbf{2}}^{f}-\chi_{\mathbf{2}}^{r}\right)}{\prod_{i \neq j}\left(1-t^{2} w_{i} w_{j}^{-1}\right)}\left(\chi_{\mathbf{7 5 6}}^{\mathrm{SU}(4)}+\left(2-\chi_{\mathbf{3}}^{r}\right) \chi_{\mathbf{5 4 0}}^{\mathrm{SU}(4)}+\left(-2 \chi_{\mathbf{3}}^{r}+\chi_{\mathbf{5}}^{r}+4\right) \chi_{\mathbf{3 6 0}}^{\mathrm{SU}(4)}-\chi_{\mathbf{3}}^{r} \chi_{\mathbf{2 8 0}}^{\mathrm{SU}(4)}\right. \\
& +\left(-\chi_{\mathbf{2}}^{f} \chi_{\mathbf{2}}^{r}-2 \chi_{\mathbf{3}}^{r}+1\right) \chi_{\mathbf{2 2 4}}^{\mathrm{SU}(4)}+\left(-\chi_{\mathbf{2}}^{f} \chi_{\mathbf{2}}^{r}-3 \chi_{\mathbf{3}}^{r}+\chi_{\mathbf{5}}^{r}+2\right) \chi_{\mathbf{1 6 0}}^{\mathrm{SU}(4)}+\left(\chi_{\mathbf{2}}^{f} \chi_{\mathbf{4}}^{r}-2 \chi_{\mathbf{3}}^{r}+2 \chi_{\mathbf{5}}^{r}+3\right) \chi_{\mathbf{1 4 0} \mathbf{0}^{\prime}}^{\mathrm{SU}(4)} \\
& +\left(-\chi_{\mathbf{2}}^{f} \chi_{\mathbf{2}}^{r}+2 \chi_{\mathbf{2}}^{f} \chi_{\mathbf{4}}^{r}-\chi_{\mathbf{2}}^{f} \chi_{\mathbf{6}}^{r}+\chi_{\mathbf{3}}^{f}-5 \chi_{\mathbf{3}}^{r}+3 \chi_{\mathbf{5}}^{r}-\chi_{\mathbf{7}}^{r}+7\right) \chi_{\mathbf{1 4 0}}^{\mathrm{SU}(4)}+\left(\chi_{\mathbf{2}}^{f} \chi_{\mathbf{4}}^{r}+\chi_{\mathbf{5}}^{r}+2\right) \chi_{\mathbf{1 2 0}}^{\mathrm{SU}(4)} \\
& +\left(\chi_{\mathbf{2}}^{f} \chi_{\mathbf{4}}^{r}-\chi_{\mathbf{3}}^{r}+\chi_{\mathbf{5}}^{r}-\chi_{\mathbf{7}}^{r}+3\right) \chi_{\mathbf{8 4}}^{\mathrm{SU}(4)}+\left(-2 \chi_{\mathbf{2}}^{f} \chi_{\mathbf{2}}^{r}-\chi_{\mathbf{3}}^{f} \chi_{\mathbf{3}}^{r}-2 \chi_{\mathbf{2}}^{f} \chi_{\mathbf{6}}^{r}-5 \chi_{\mathbf{3}}^{r}+\chi_{\mathbf{5}}^{r}-2 \chi_{\mathbf{7}}^{r}+5\right) \chi_{\mathbf{6 0}}^{\mathrm{SU}(4)} \\
& +\left(-\chi_{\mathbf{2}}^{f} \chi_{\mathbf{2}}^{r}+2 \chi_{\mathbf{2}}^{f} \chi_{\mathbf{4}}^{r}-\chi_{\mathbf{2}}^{f} \chi_{\mathbf{6}}^{r}+\chi_{\mathbf{2}}^{f} \chi_{\mathbf{8}}^{r}+\chi_{\mathbf{3}}^{f}-4 \chi_{\mathbf{3}}^{r}+3 \chi_{\mathbf{5}}^{r}+\chi_{\mathbf{9}}^{r}+5\right) \chi_{\mathbf{3 6}}^{\mathrm{SU}(4)}+\left(-2 \chi_{\mathbf{2}}^{f} \chi_{\mathbf{2}}^{r}-\chi_{\mathbf{3}}^{f} \chi_{\mathbf{3}}^{r}-\chi_{\mathbf{2}}^{f} \chi_{\mathbf{4}}^{r}\right. \\
& \left.-\chi_{\mathbf{3}}^{f} \chi_{\mathbf{5}}^{r}-2 \chi_{\mathbf{2}}^{f} \chi_{\mathbf{6}}^{r}-3 \chi_{\mathbf{3}}^{r}-\chi_{\mathbf{5}}^{r}-2 \chi_{\mathbf{7}}^{r}+2\right) \chi_{\mathbf{2 0}}^{\mathrm{SU}(4)}+\left(\chi_{\mathbf{3}}^{f} \chi_{\mathbf{5}}^{r}+\chi_{\mathbf{3}}^{f} \chi_{\mathbf{7}}^{r}+4 \chi_{\mathbf{2}}^{f} \chi_{\mathbf{4}}^{r}+2 \chi_{\mathbf{2}}^{f} \chi_{\mathbf{8}}^{r}+\chi_{\mathbf{3}}^{f}-2 \chi_{\mathbf{3}}^{r}+4 \chi_{\mathbf{5}}^{r}\right. \\
& \left.+\chi_{\mathbf{9}}^{r}+6\right) \chi_{\mathbf{2 0}}^{\mathrm{SU}(4)}+\left(-2 \chi_{\mathbf{2}}^{f} \chi_{\mathbf{2}}^{r}-\chi_{\mathbf{3}}^{f} \chi_{\mathbf{3}}^{r}-\chi_{\mathbf{2}}^{f} \chi_{\mathbf{4}}^{r}-\chi_{\mathbf{3}}^{f} \chi_{\mathbf{5}}^{r}-3 \chi_{\mathbf{2}}^{f} \chi_{\mathbf{6}}^{r}-\chi_{\mathbf{3}}^{f} \chi_{\mathbf{7}}^{r}-\chi_{\mathbf{2}}^{f} \chi_{\mathbf{8}}^{r}-\chi_{\mathbf{3}}^{f} \chi_{\mathbf{9}}^{r}-\chi_{\mathbf{2}}^{f} \chi_{\mathbf{1 0}}^{r}\right. \\
& \left.\left.-4 \chi_{\mathbf{3}}^{r}-\chi_{\mathbf{5}}^{r}-2 \chi_{\mathbf{7}}^{r}-\chi_{\mathbf{9}}^{r}-\chi^{r}{ }_{11}+1\right) \chi_{\mathbf{4}}^{\mathrm{SU}(4)}\right)+\mathcal{O}\left(q^{2}\right) \\
& \frac{\mathcal{W}_{\mathbf{6}}^{\mathrm{U}(4)}}{\mathcal{W}_{\mathbf{1}}^{\mathrm{U}(4)}}=\chi_{\mathbf{6}}^{\mathrm{SU}(4)}+q \cdot \frac{t^{12} \cdot\left(\chi_{\mathbf{2}}^{f}-\chi_{\mathbf{2}}^{l}\right)\left(\chi_{\mathbf{2}}^{f}-\chi_{\mathbf{2}}^{r}\right)}{\prod_{i \neq j}\left(1-t^{2} w_{i} w_{j}^{-1}\right)}\left(\chi_{\mathbf{9 6 0}}^{\mathrm{SU}(4)}+\left(2-\chi_{\mathbf{3}}^{r}\right)\left(\chi_{\mathbf{6 3 0}}^{\mathrm{SU}(4)}+\chi_{\overline{\mathbf{6 3 0}}}^{\mathrm{SU}(4)}\right)+\left(\chi_{\mathbf{5}}^{r}-3 \chi_{\mathbf{3}}^{r}+4\right) \chi_{\mathbf{3 8 4}}^{\mathrm{SU}(4)}\right. \\
& +\left(\chi_{\mathbf{3}}^{f}-5 \chi_{\mathbf{3}}^{r}+2 \chi_{\mathbf{2}}^{f} \chi_{\mathbf{4}}^{r}+4 \chi_{\mathbf{5}}^{r}+10\right) \chi_{\mathbf{3 0 0}}^{\mathrm{SU}(4)}-\left(\chi_{\mathbf{2}}^{f} \chi_{\mathbf{2}}^{r}+2 \chi_{\mathbf{3}}^{r}-1\right)\left(\chi_{\mathbf{2 7 0}}^{\mathrm{SU}(4)}+\chi_{\mathbf{2} \overline{\mathbf{7}} \mathbf{0}}^{\mathrm{SU}(4)}\right)+\left(\chi_{\mathbf{2}}^{f} \chi_{\mathbf{4}}^{r}+\chi_{\mathbf{5}}^{r}+2\right) \\
& \times\left(\chi_{\mathbf{1 4 0}^{\prime \prime}}^{\mathrm{SU}(4)}+\chi_{\mathbf{1 4 0}^{\prime \prime}}^{\mathrm{SU}(4)}\right)-\left(3 \chi_{\mathbf{2}}^{f} \chi_{\mathbf{2}}^{r}+\chi_{\mathbf{3}}^{f} \chi_{\mathbf{3}}^{r}+7 \chi_{\mathbf{3}}^{r}-2 \chi_{\mathbf{5}}^{r}+\chi_{\mathbf{2}}^{f} \chi_{\mathbf{6}}^{r}+\chi_{\mathbf{7}}^{r}-6\right)\left(\chi_{\mathbf{1 2 6}}^{\mathrm{SU}(4)}+\chi_{\mathbf{1} \mathbf{2} \mathbf{6}}^{\mathrm{SU}(4)}\right)+\left(\chi_{\mathbf{3}}^{f}\right. \\
& \left.-\chi_{\mathbf{2}}^{f} \chi_{\mathbf{2}}^{r}-5 \chi_{\mathbf{3}}^{r}+2 \chi_{\mathbf{2}}^{f} \chi_{\mathbf{4}}^{r}+2 \chi_{\mathbf{5}}^{r}-2 \chi_{\mathbf{2}}^{f} \chi_{\mathbf{6}}^{r}-2 \chi_{\mathbf{7}}^{r}+7\right)\left(\chi_{\mathbf{7 0}}^{\mathrm{SU}(4)}+\chi_{\mathbf{7 0}}^{\mathrm{SU}(4)}\right)+\left(-2 \chi_{\mathbf{3}}^{r} \chi_{\mathbf{3}}^{f}+2 \chi_{\mathbf{3}}^{f}-4 \chi_{\mathbf{2}}^{f} \chi_{\mathbf{2}}^{r}\right. \\
& \left.-12 \chi_{\mathbf{3}}^{r}+4 \chi_{\mathbf{2}}^{f} \chi_{\mathbf{4}}^{r}+6 \chi_{\mathbf{5}}^{r}-4 \chi_{\mathbf{2}}^{f} \chi_{\mathbf{6}}^{r}-3 \chi_{\mathbf{7}}^{r}+2 \chi_{\mathbf{2}}^{f} \chi_{\mathbf{8}}^{r}+\chi_{\mathbf{9}}^{r}+15\right) \chi_{\mathbf{6 4}}^{\mathrm{SU}(4)}+\left(\chi_{\mathbf{3}}^{r} \chi_{\mathbf{3}}^{f}+\chi_{\mathbf{5}}^{r} \chi_{\mathbf{3}}^{f}+\chi_{\mathbf{3}}^{f}+2 \chi_{\mathbf{2}}^{f} \chi_{\mathbf{2}}^{r}\right. \\
& \left.-\chi_{\mathbf{3}}^{r}+4 \chi_{\mathbf{2}}^{f} \chi_{\mathbf{4}}^{r}+4 \chi_{\mathbf{5}}^{r}-\chi_{\mathbf{7}}^{r}+7\right) \chi_{\mathbf{5 0}}^{\mathrm{SU}(4)}+\left(\chi_{\mathbf{5}}^{r} \chi_{\mathbf{3}}^{f}+\chi_{\mathbf{7}}^{r} \chi_{\mathbf{3}}^{f}+\chi_{\mathbf{3}}^{f}-\chi_{\mathbf{2}}^{f} \chi_{\mathbf{2}}^{r}-3 \chi_{\mathbf{3}}^{r}+4 \chi_{\mathbf{2}}^{f} \chi_{\mathbf{4}}^{r}+5 \chi_{\mathbf{5}}^{r}+\chi_{\mathbf{2}}^{f} \chi_{\mathbf{6}}^{r}\right. \\
& \left.+2 \chi_{\mathbf{7}}^{r}+3 \chi_{\mathbf{2}}^{f} \chi_{\mathbf{8}}^{r}+2 \chi_{\mathbf{9}}^{r}+5\right)\left(\chi_{\mathbf{1 0}}^{\mathrm{SU}(4)}+\chi_{\mathbf{1 0}}^{\mathrm{SU}(4)}\right)-\left(5 \chi_{\mathbf{3}}^{r}+\chi_{\mathbf{5}}^{r}+5 \chi_{\mathbf{7}}^{r}+2 \chi_{\mathbf{9}}^{r}+\chi_{\mathbf{3}}^{f}\left(\chi_{\mathbf{3}}^{r}+\chi_{\mathbf{5}}^{r}+\chi_{\mathbf{7}}^{r}+\chi_{\mathbf{9}}^{r}-1\right)\right. \\
& \left.\left.+2 \chi_{\mathbf{2}}^{f}\left(\chi_{\mathbf{2}}^{r}+3 \chi_{\mathbf{6}}^{r}+\chi_{\mathbf{8}}^{r}+\chi_{\mathbf{1 0}}^{r}\right)+\chi_{\mathbf{1 1}}^{r}-5\right) \chi_{\mathbf{6}}^{\mathrm{SU}(4)}\right)+\mathcal{O}\left(q^{2}\right) .
\end{aligned}
$$

The Wilson loops for all the other minuscule representations can be found from the above ones, by using the rule $\mathcal{W}_{\bigwedge^{p}}^{\mathrm{U}(N)}=\mathcal{W}_{\Lambda^{N-p}}^{\mathrm{U}(N)}\left(w_{i} \rightarrow w_{i}^{-1}\right)$, i.e. by conjugating the $\mathrm{U}(1)^{N}$ electric charges. Upon suppressing the $q$ correction for the bulk factor $\mathcal{W}^{\mathrm{U}\left(1^{\prime}\right)}$ in (2.29), one can finally obtain the $5 \mathrm{~d} / 1 \mathrm{~d}$ QFT partition functions as follows.

$$
\begin{aligned}
& Z_{5 \mathrm{~d} / 1 \mathrm{~d}}^{(2,1)}=\left(x_{1}+x_{1}^{-1}\right) \mathcal{W}_{\mathbf{1}}^{\mathrm{U}(2)}-x_{1}^{0} \mathcal{W}_{\mathbf{2}}^{\mathrm{U}(2)}, \quad Z_{5 \mathrm{~d} / 1 \mathrm{~d}}^{(3,1)}=\left(x_{1}^{3 / 2}-x_{1}^{-3 / 2}\right) \mathcal{W}_{\mathbf{1}}^{\mathrm{U}(3)}+x_{1}^{-1 / 2} \mathcal{W}_{\overline{\mathbf{3}}}^{\mathrm{U}(3)}-x_{1}^{1 / 2} \mathcal{W}_{\mathbf{3}}^{\mathrm{U}(3)}, \\
& Z_{5 \mathrm{~d} / 1 \mathrm{~d}}^{(4,1)}=\left(x_{1}^{-2}+x_{1}^{2}\right) \mathcal{W}_{\mathbf{1}}^{\mathrm{U}(4)}-x_{1}^{-1} \mathcal{W}_{\overline{\mathbf{4}}}^{\mathrm{U}(4)}+x_{1}^{0} \mathcal{W}_{\mathbf{6}}^{\mathrm{U}(4)}-x_{1}^{1} \mathcal{W}_{\mathbf{4}}^{\mathrm{U}(4)}
\end{aligned}
$$

Multiple D4'-branes are needed for Wilson loops in more general representations. For example, the $\mathrm{U}(2)$ symmetric Wilson loop $\mathcal{W}_{\mathbf{3}}^{\mathrm{U}(2)}$ appears in the case of $N=N^{\prime}=2$, for which we expect

$$
Z_{\mathrm{ADHM}}^{(2,2)}=-2 \mathcal{W}_{\mathbf{2}}^{\mathrm{U}(2)} \mathcal{W}_{\mathbf{2}}^{\mathrm{U}\left(2^{\prime}\right)}+\mathcal{W}_{\mathbf{1}}^{\mathrm{U}(2)}\left(\mathcal{W}_{\mathbf{3}}^{\mathrm{U}\left(2^{\prime}\right)}+\mathcal{W}_{\mathbf{1}}^{\mathrm{U}\left(2^{\prime}\right)}\right)+\left(\mathcal{W}_{\mathbf{3}}^{\mathrm{U}(2)}+\mathcal{W}_{\mathbf{1}}^{\mathrm{U}(2)}\right) \mathcal{W}_{\mathbf{1}}^{\mathrm{U}\left(2^{\prime}\right)}
$$

Substituting the previously evaluated $\mathcal{W}_{2}^{\mathrm{U}(2)}$ and its bulk analogue, the combination $\mathcal{W}_{\mathbf{3}}^{\mathrm{U}(2)} \mathcal{W}_{\mathbf{1}}^{\mathrm{U}\left(2^{\prime}\right)}+\mathcal{W}_{\mathbf{1}}^{\mathrm{U}(2)} \mathcal{W}_{\mathbf{3}}^{\mathrm{U}\left(2^{\prime}\right)}$ can be found. The two summands in this combination can be 
disentangled from each other by using the following observations which will also be useful for similar issues that will arise with generic $N$ and $N^{\prime}:{ }^{3}$

- The $k$-instanton correction of the Wilson lines takes the form of [36-38]:

$$
\left.\mathcal{W}_{\mathbf{R}}^{\mathrm{U}(N)}\right|_{q^{k}}=\frac{\sum_{\mathbf{r}} c_{\mathbf{r}}\left(\epsilon_{1,2}, m\right) \cdot \chi_{\mathbf{r}}^{\mathrm{SU}(N)}\left(\alpha_{a}\right)}{\prod_{n=1}^{k} \operatorname{sh}\left(n \epsilon_{1}\right) \operatorname{sh}\left(n \epsilon_{2}\right) \cdot \prod_{n, m>0}^{n m \leq k} \prod_{a \neq b} \operatorname{sh}\left(n \epsilon_{1}+m \epsilon_{2}+\alpha_{a}-\alpha_{b}\right)}
$$

where $\mathbf{r}$ runs over all $\mathrm{SU}(N)$ irreducible representations that appear in $\mathbf{R} \otimes \operatorname{adj}^{\ell(N, k)}$. The positive integer $\ell(N, k)$ can be determined by inspecting the $k$-instanton partition function $\left.\mathcal{W}_{\mathbf{1}}^{\mathrm{U}(N)}\right|_{q^{k}}$.

- $\mathcal{W}_{\mathbf{R}}^{\mathrm{U}(N)}$ and $\mathcal{W}_{\mathbf{R}^{\prime}}^{\mathrm{U}\left(N^{\prime}\right)}$ with $N=N^{\prime}$ and $\mathbf{R}=\mathbf{R}^{\prime}$ must have the same functional form up to the fugacity exchange (2.16).

- The series expansion of the normalized Wilson line $\mathcal{W}_{\mathbf{R}}^{\mathrm{U}(N)} /\left.\mathcal{W}_{\mathbf{1}}^{\mathrm{U}(N)}\right|_{q^{k}}$ in the $\mathrm{SU}(N)$ simple roots $\left\{\mathfrak{n}_{i} \equiv w_{i} / w_{i+1} \mid 1 \leq i \leq N-1\right\}$ starts with the 'leading' states having the following form:

$$
\prod_{i=1}^{N-1} \mathfrak{n}_{i}^{l_{i}} \quad \text { with }\left(l_{1}, l_{2}, \cdots, l_{N-1}\right)=\left(\lambda_{1}, \lambda_{2}, \cdots, \lambda_{N-1}\right)+\left(\beta_{1}, \beta_{2}, \cdots, \beta_{N-1}\right) \in \mathbf{R}
$$

where $\left(\lambda_{1}, \lambda_{2}, \cdots, \lambda_{N-1}\right)$ is the lowest weight of the representation $\mathbf{R}$ in $\alpha$-basis, $\left(\beta_{1}, \beta_{2}, \cdots, \beta_{N-1}\right)$ can be any $\mathrm{SU}(N)$ positive ( not just simple) root. The coefficients of these leading states carry $\mathrm{SU}(2)_{r} \subset \mathrm{SU}(2)_{1 R} \times \mathrm{SU}(2)_{2 R}$ spin, which is bounded as $2 j_{r} \leq k$. More generally, the coefficients of the 'subleading' states, having the form of $\prod_{i=1}^{N-1} \mathfrak{n}_{i}^{l_{i}} \cdot \mathfrak{n}_{j}^{\ell}$ and not overlapping with the set of 'leading' states (2.37), are subject to the following $\mathrm{SU}(2)_{r}$ spin bound condition:

$$
2 j_{r} \leq k+(k+1) \ell
$$

We utilized the above observations and computed the Wilson loop expectation value $\mathcal{W}_{\mathbf{3}}^{\mathrm{U}(2)}$ up to 2 instanton corrections. For brevity, we explicitly show $\mathcal{W}_{\mathbf{3}}^{\mathrm{U}(2)} / \mathcal{W}_{\mathbf{1}}^{\mathrm{U}(2)}$ only up to the 1-instanton order:

$\frac{\mathcal{W}_{\mathbf{3}}^{\mathrm{U}(2)}}{\mathcal{W}_{\mathbf{1}}^{\mathrm{U}(2)}}=\chi_{\mathbf{3}}^{\mathrm{SU}(2)}+q \cdot \frac{t^{2} \cdot\left(\chi_{\mathbf{2}}^{f}-\chi_{\mathbf{2}}^{l}\right)}{\left(1-t^{2} w_{1}^{ \pm 2}\right)} \cdot\left(\chi_{\mathbf{3}}^{\mathrm{SU}(2)}\left(-\chi_{\mathbf{2}}^{f}+\chi_{\mathbf{2}}^{l}+\chi_{\mathbf{2}}^{r}\right)-\chi_{\mathbf{2}}^{f} \chi_{\mathbf{2}}^{l} \chi_{\mathbf{2}}^{r}-\chi_{\mathbf{2}}^{f}+\chi_{\mathbf{2}}^{l}+\chi_{\mathbf{2}}^{r}\right)+\mathcal{O}\left(q^{2}\right)$

\footnotetext{
${ }^{3}$ These observations are based on the $\mathrm{SU}(2), \mathrm{SU}(3)$, SU(4) minuscule Wilson loops with 2 instanton corrections.
} 
The same procedure can be repeated for $\left(N, N^{\prime}\right)=(2,3)$ and $\left(N, N^{\prime}\right)=(3,2)$, for which we have

$$
\begin{aligned}
Z_{\mathrm{ADHM}}^{(3,2)}= & \left(\mathcal{W}_{\mathbf{6}}^{\mathrm{U}(3)}+\mathcal{W}_{\overline{\mathbf{6}}}^{\mathrm{U}(3)}+2 \mathcal{W}_{\mathbf{1}}^{\mathrm{U}(3)}\right) \mathcal{W}_{\mathbf{1}}^{\mathrm{U}\left(2^{\prime}\right)}-\left(\mathcal{W}_{\mathbf{8}}^{\mathrm{U}(3)}+\mathcal{W}_{\mathbf{3}}^{\mathrm{U}(3)}+\mathcal{W}_{\overline{\mathbf{3}}}^{\mathrm{U}(3)}\right) \mathcal{W}_{\mathbf{2}}^{\mathrm{U}\left(2^{\prime}\right)} \\
& +\left(\mathcal{W}_{\mathbf{3}}^{\mathrm{U}(3)}+\mathcal{W}_{\overline{\mathbf{3}}}^{\mathrm{U}(3)}\right) \mathcal{W}_{\mathbf{3}}^{\mathrm{U}\left(2^{\prime}\right)}-\mathcal{W}_{\mathbf{1}}^{\mathrm{U}(3)} \mathcal{W}_{\mathbf{4}}^{\mathrm{U}\left(2^{\prime}\right)} \\
Z_{\mathrm{ADHM}}^{(2,3)}= & \mathcal{W}_{\mathbf{1}}^{\mathrm{U}(2)}\left(\mathcal{W}_{\mathbf{6}}^{\mathrm{U}\left(3^{\prime}\right)}+\mathcal{W}_{\overline{\mathbf{6}}}^{\mathrm{U}\left(3^{\prime}\right)}+2 \mathcal{W}_{\mathbf{1}}^{\mathrm{U}\left(3^{\prime}\right)}\right)-\mathcal{W}_{\mathbf{2}}^{\mathrm{U}(2)}\left(\mathcal{W}_{\mathbf{8}}^{\mathrm{U}\left(3^{\prime}\right)}+\mathcal{W}_{\mathbf{3}}^{\mathrm{U}\left(3^{\prime}\right)}+\mathcal{W}_{\overline{\mathbf{3}}}^{\mathrm{U}\left(3^{\prime}\right)}\right) \\
& +\mathcal{W}_{\mathbf{3}}^{\mathrm{U}(2)}\left(\mathcal{W}_{\mathbf{3}}^{\mathrm{U}\left(3^{\prime}\right)}+\mathcal{W}_{\overline{\mathbf{3}}}^{\mathrm{U}\left(3^{\prime}\right)}\right)-\mathcal{W}_{\mathbf{4}}^{\mathrm{U}(2)} \mathcal{W}_{\mathbf{1}}^{\mathrm{U}\left(3^{\prime}\right)}
\end{aligned}
$$

Similarly we found the Wilson lines $\mathcal{W}_{\mathbf{4}}^{\mathrm{U}(2)}, \mathcal{W}_{\mathbf{6}}^{\mathrm{U}(3)}, \mathcal{W}_{\overline{\mathbf{6}}}^{\mathrm{U}(3)}=\left(\mathcal{W}_{\mathbf{6}}^{\mathrm{U}(3)}\right)^{*}, \mathcal{W}_{\mathbf{8}}^{\mathrm{U}(3)}$ up to 1 instanton order.

$$
\begin{aligned}
& \frac{\mathcal{W}_{\mathbf{4}}^{\mathrm{U}(2)}}{\mathcal{W}_{\mathbf{1}}^{\mathrm{U}(2)}}=\chi_{\mathbf{4}}^{\mathrm{SU}(3)}-q \cdot \frac{t^{2} \cdot\left(\chi_{\mathbf{2}}^{f}-\chi_{\mathbf{2}}^{l}\right)}{\left(1-t^{2} w_{1}^{ \pm 2}\right)}\left(\chi_{\mathbf{4}}^{\mathrm{SU}(2)}\left(\chi_{\mathbf{2}}^{f}+\chi_{\mathbf{2}}^{l}+\chi_{\mathbf{2}}^{r}\right)\right. \\
& \left.+\chi_{\mathbf{2}}^{\mathrm{SU}(2)}\left(-2 \chi_{\mathbf{4}}^{r}+\chi_{\mathbf{2}}^{f} \chi_{\mathbf{2}}^{l} \chi_{\mathbf{2}}^{r}-\chi_{\mathbf{3}}^{l} \chi_{\mathbf{2}}^{r}-2 \chi_{\mathbf{2}}^{l} \chi_{\mathbf{3}}^{r}+\chi_{\mathbf{2}}^{f} \chi_{\mathbf{3}}^{l}-\chi_{\mathbf{2}}^{r}+\chi_{\mathbf{2}}^{f}\right)\right)+\mathcal{O}\left(q^{2}\right) \\
& \frac{\mathcal{W}_{\mathbf{6}}^{\mathrm{U}(3)}}{\mathcal{W}_{\mathbf{1}}^{\mathrm{U}(3)}}=\chi_{\mathbf{6}}^{\mathrm{SU}(3)}+q \cdot \frac{t^{6} \cdot\left(\chi_{\mathbf{2}}^{f}-\chi_{\mathbf{2}}^{l}\right)}{\prod_{i \neq j}^{3}\left(1-t^{2} w_{i} w_{j}^{-1}\right)}\left(\left(\chi_{\mathbf{2}}^{l}+\chi_{\mathbf{2}}^{r}-\chi_{\mathbf{2}}^{f}\right) \chi_{\mathbf{4 2}}^{\mathrm{SU}(3)}+\left(\chi_{\mathbf{2}}^{f} \chi_{\mathbf{3}}^{r}-2 \chi_{\mathbf{2}}^{f}-\chi_{\mathbf{2}}^{l} \chi_{\mathbf{3}}^{r}+\chi_{\mathbf{2}}^{l}+\chi_{\mathbf{2}}^{r}-\chi_{\mathbf{4}}^{r}\right) \chi_{\mathbf{2 4}}^{\mathrm{SU}(3)}\right. \\
& +\left(\chi_{\mathbf{2}}^{r}-\chi_{\mathbf{2}}^{l} \chi_{\mathbf{2}}^{f} \chi_{\mathbf{2}}^{r}-\chi_{\mathbf{2}}^{f}+\chi_{\mathbf{2}}^{l}\right) \chi_{\overline{\mathbf{1 5}}^{\prime}}^{\mathrm{SU}(3)}+\left(\chi_{\mathbf{2}}^{f} \chi_{\mathbf{2}}^{l} \chi_{\mathbf{4}}^{r}-\chi_{\mathbf{2}}^{l} \chi_{\mathbf{2}}^{f} \chi_{\mathbf{2}}^{r}+\chi_{\mathbf{3}}^{f} \chi_{\mathbf{2}}^{l}+2 \chi_{\mathbf{2}}^{f} \chi_{\mathbf{3}}^{r}+\chi_{\mathbf{3}}^{f} \chi_{\mathbf{2}}^{r}-5 \chi_{\mathbf{2}}^{f}-2 \chi_{\mathbf{2}}^{l} \chi_{\mathbf{3}}^{r}\right. \\
& \left.+3 \chi_{\mathbf{2}}^{l}+2 \chi_{\mathbf{2}}^{r}-3 \chi_{\mathbf{4}}^{r}\right) \chi_{\mathbf{1 5}}^{\mathrm{SU}(3)}+\left(-\chi_{\mathbf{2}}^{l} \chi_{\mathbf{2}}^{f} \chi_{\mathbf{2}}^{r}+\chi_{\mathbf{2}}^{f} \chi_{\mathbf{2}}^{l} \chi_{\mathbf{4}}^{r}-\chi_{\mathbf{3}}^{f} \chi_{\mathbf{2}}^{l} \chi_{\mathbf{3}}^{r}+2 \chi_{\mathbf{2}}^{f} \chi_{\mathbf{3}}^{r}-\chi_{\mathbf{3}}^{f} \chi_{\mathbf{4}}^{r}-4 \chi_{\mathbf{2}}^{f}+\chi_{\mathbf{2}}^{l} \chi_{\mathbf{5}}^{r}+3 \chi_{\mathbf{2}}^{l}\right. \\
& \left.+3 \chi_{\mathbf{2}}^{r}-\chi_{\mathbf{4}}^{r}+\chi_{\mathbf{6}}^{r}\right) \chi_{\mathbf{6}}^{\mathrm{SU}(3)}+\left(-\chi_{\mathbf{2}}^{l} \chi_{\mathbf{2}}^{r}+\chi_{\mathbf{2}}^{f} \chi_{\mathbf{2}}^{l} \chi_{\mathbf{4}}^{r}-\chi_{\mathbf{2}}^{f} \chi_{\mathbf{2}}^{l} \chi_{\mathbf{6}}^{r}+\chi_{\mathbf{3}}^{f} \chi_{\mathbf{2}}^{l}+2 \chi_{\mathbf{2}}^{f} \chi_{\mathbf{3}}^{r}+\chi_{\mathbf{3}}^{f} \chi_{\mathbf{2}}^{r}-\chi_{\mathbf{3}}^{f} \chi_{\mathbf{4}}^{r}-4 \chi_{\mathbf{2}}^{f}\right. \\
& \left.\left.-2 \chi_{\mathbf{2}}^{l} \chi_{\mathbf{3}}^{r}+2 \chi_{\mathbf{2}}^{l}+2 \chi_{\mathbf{2}}^{r}-2 \chi_{\mathbf{4}}^{r}+\chi_{\mathbf{6}}^{r}\right) \chi_{\mathbf{3}}^{\mathrm{SU}(3)}\right)+\mathcal{O}\left(q^{2}\right) \\
& \frac{\mathcal{W}_{\mathbf{8}}^{\mathrm{U}(3)}}{\mathcal{W}_{\mathbf{1}}^{\mathrm{U}(3)}}=\chi_{\mathbf{8}}^{\mathrm{SU}(3)}+q \cdot \frac{t^{6} \cdot\left(\chi_{\mathbf{2}}^{f}-\chi_{\mathbf{2}}^{l}\right)}{\prod_{i \neq j}^{3}\left(1-t^{2} w_{i} w_{j}^{-1}\right)}\left(\left(\chi_{\mathbf{2}}^{r}-\chi_{\mathbf{2}}^{f}\right)\left(\chi_{\mathbf{3 5}}^{\mathrm{SU}(3)}+\chi_{\overline{\mathbf{3 5}}}^{\mathrm{SU}(3)}\right)+\left(2 \chi_{\mathbf{2}}^{f} \chi_{\mathbf{3}}^{r}-7 \chi_{\mathbf{2}}^{f}+2 \chi_{\mathbf{2}}^{l}-2 \chi_{\mathbf{4}}^{r}\right) \chi_{\mathbf{2} \mathbf{7}}^{\mathrm{SU}(3)}\right. \\
& +\left(4 \chi_{\mathbf{2}}^{r}-\chi_{\mathbf{2}}^{l} \chi_{\mathbf{2}}^{f} \chi_{\mathbf{2}}^{r}+4 \chi_{\mathbf{2}}^{f} \chi_{\mathbf{3}}^{r}+\chi_{\mathbf{3}}^{f} \chi_{\mathbf{2}}^{r}-4 \chi_{\mathbf{2}}^{f}-\chi_{\mathbf{2}}^{l} \chi_{\mathbf{3}}^{r}+\chi_{\mathbf{2}}^{l}\right)\left(\chi_{\mathbf{1 0}}^{\mathrm{SU}(3)}+\chi_{\overline{\mathbf{1 0}}}^{\mathrm{SU}(3)}\right)+\left(2 \chi_{\mathbf{2}}^{f} \chi_{\mathbf{2}}^{l} \chi_{\mathbf{4}}^{r}-\chi_{\mathbf{2}}^{l} \chi_{\mathbf{2}}^{f} \chi_{\mathbf{2}}^{r}\right. \\
& \left.+\chi_{\mathbf{3}}^{f} \chi_{\mathbf{2}}^{l}+5 \chi_{\mathbf{2}}^{f} \chi_{\mathbf{3}}^{r}-3 \chi_{\mathbf{2}}^{f} \chi_{\mathbf{5}}^{r}+\chi_{\mathbf{3}}^{f} \chi_{\mathbf{2}}^{r}-2 \chi_{\mathbf{3}}^{f} \chi_{\mathbf{4}}^{r}-12 \chi_{\mathbf{2}}^{f}-2 \chi_{\mathbf{2}}^{l} \chi_{\mathbf{3}}^{r}+3 \chi_{\mathbf{2}}^{l}+3 \chi_{\mathbf{2}}^{r}-6 \chi_{\mathbf{4}}^{r}\right) \chi_{\mathbf{8}}^{\mathrm{SU}(3)}-\chi_{\mathbf{2}}^{l} \chi_{\mathbf{2}}^{f} \chi_{\mathbf{2}}^{r} \\
& -\chi_{2}^{f} \chi_{2}^{l} \chi_{\mathbf{6}}^{r}-\chi_{3}^{f} \chi_{2}^{l} \chi_{3}^{r}-\chi_{3}^{f} \chi_{2}^{l} \chi_{5}^{r}+4 \chi_{2}^{f} \chi_{3}^{r}+2 \chi_{2}^{f} \chi_{5}^{r}+2 \chi_{2}^{f} \chi_{7}^{r}+\chi_{3}^{f} \chi_{2}^{r}+\chi_{3}^{f} \chi_{\mathbf{6}}^{r}-3 \chi_{\mathbf{2}}^{f}+\chi_{2}^{l} \chi_{3}^{r}+\chi_{2}^{l} \chi_{5}^{r}+2 \chi_{2}^{l} \\
& \left.+3 \chi_{\mathbf{2}}^{r}+2 \chi_{\mathbf{4}}^{r}+5 \chi_{\mathbf{6}}^{r}+2 \chi_{\mathbf{8}}^{r}\right)+\mathcal{O}\left(q^{2}\right) \text {. }
\end{aligned}
$$

These results can be combined into the line defect partition function for $\mathrm{U}(2)$ and $\mathrm{U}(3)$ gauge theories.

Finally, the complete 5d BPS spectrum with a line defect can be obtained by multiplying the $5 \mathrm{~d} / 1 \mathrm{~d}$ partition function of (2.28) with the perturbative W-bosons' partition function $Z_{\text {pert }}$. This perturbative piece can be computed using the equivariant index theorem [39] or equivalently by counting the BPS letter operators, and is given as

$Z_{\text {pert }}^{\mathrm{U}(N)}=\operatorname{PE}\left[\frac{\operatorname{sh}\left(M \pm \epsilon_{+}\right)}{\operatorname{sh}\left(\epsilon_{+} \pm \epsilon_{-}\right)} \cdot\left(\chi_{\mathbf{a d j}}^{\mathrm{SU}(N)}+1\right)^{+}\right]=\operatorname{PE}\left[\frac{t\left(\mu+\mu^{-1}-t-t^{-1}\right)}{(1-t u)\left(1-t u^{-1}\right)} \cdot\left(\chi_{\mathbf{a d j}}^{\mathrm{SU}(N)}+1\right)^{+}\right]$

The superscript ' + ' means those non-BPS states corresponding to non-positive roots are all discarded. 


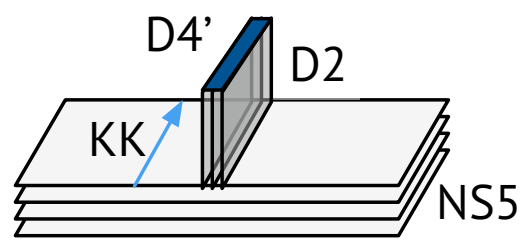

Figure 3. The brane configuration describing codimension-4 Wilson surfaces in $6 \mathrm{~d}(2,0)$ theories.

\begin{tabular}{|c|cccccccccc|}
\hline & 0 & 1 & 2 & 3 & 4 & 5 & 6 & 7 & 8 & 9 \\
\hline$N$ NS5 & $\bullet$ & $\bullet$ & $\bullet$ & $\bullet$ & $\bullet$ & $\bullet$ & & & & \\
$N^{\prime}$ D4 & $\bullet$ & $\bullet$ & & & & & $\bullet$ & $\bullet$ & $\bullet$ & \\
1 D6 & $\bullet$ & $\bullet$ & $\bullet$ & $\bullet$ & $\bullet$ & $\bullet$ & & & & $\bullet$ \\
D2 & $\bullet$ & $\bullet$ & & & & & & & & $\bullet$ \\
\hline
\end{tabular}

Table 3. Brane configuration for $6 \mathrm{~d} \mathcal{N}=(2,0)$ SCFTs with Wilson surface defects.

\section{Wilson surfaces in $6 \mathrm{~d} \mathcal{N}=(2,0)$ SCFTs}

\subsection{Gauge theories on self-dual strings}

In order to engineer Wilson surfaces in $6 \mathrm{~d}(2,0)$ theories, we start from the D0-D4-D4' brane configuration (table 1 ) that engineers the $5 \mathrm{~d} \mathcal{N}=1^{*} \mathrm{U}(N)$ gauge theory with the $\frac{1}{2}$-BPS line defects and T-dualize along the $x^{5}$ direction, then take S-duality followed by another T-duality along $x^{5}$. The dual system is given by a type IIA configuration composed of NS5-D6-D4'-D2 branes (see table 3 as well as figure 3). The brane system preserves the $\mathrm{SO}(1,1)_{01} \times \mathrm{SO}(4)_{2345} \times \mathrm{SO}(3)_{678}$ symmetry. It is often convenient to write $\mathrm{SO}(4)_{2345} \simeq \mathrm{SU}(2)_{1 L} \times \mathrm{SU}(2)_{1 R}$ and $\mathrm{SO}(3)_{678} \simeq \mathrm{SU}(2)_{2 R}$. We denote the doublet indices of $\mathrm{SU}(2)_{1 L}, \mathrm{SU}(2)_{1 R}, \mathrm{SU}(2)_{2 R}$ by $\alpha, \dot{\alpha}, A$, respectively. The 32 supercharges of IIA string theory can be written as $Q_{ \pm \pm}^{\alpha A}$ and $Q_{ \pm \pm}^{\dot{\alpha} A}$, where the first/second subscripts are eigenvalues of $\Gamma^{01}$ and $\Gamma^{9}$. The independent SUSY projectors imposed by NS5-, D6-, D2-branes are $\Gamma^{01}, \Gamma^{2345}, \Gamma^{9}$. The presence of $\mathrm{D}^{\prime}$-branes does not yield an additional SUSY projector. The surviving supercharges are $Q_{-+}^{\dot{\alpha} A}$, satisfying $2 \mathrm{~d} \mathcal{N}=(0,4)$ supersymmetry algebra.

The parallel stack of $N$ NS5-branes engineers $6 \mathrm{~d} \mathcal{N}=(2,0)$ SCFT of $A_{N-1}$ type. We separate the NS5-branes from one another along the $x^{9}$ direction. The distances between adjacent NS5-branes are parametrized by the VEV of the tensor multiplet scalar $\Phi_{9}$. It spans the tensor branch of $(2,0)$ SCFT where the conformal symmetry is spontaneously broken. The D6-brane in table 3 is obtained from dualizing the Taub-NUT space of table 1. The BPS strings of 6d SCFTs are electric/magnetic sources of the tensor multiplets. They are realized by D2-branes suspended between an adjacent pair of NS5-branes. In the tensor branch, they acquire a non-zero tension which is proportional to the $\operatorname{VEV}\left\langle\Phi_{9}\right\rangle \neq 0$. The worldvolume gauge theory of D2-branes $[18,19]$ turns out to be useful for studying the protected quantities of $6 \mathrm{~d}$ BPS strings, such as the chiral anomaly, central charge and the elliptic genus.

The D4'-branes of table 3 are obtained from the D4'-branes of table 1 . These are located at the origin $x^{2}=\cdots=x^{5}=0$ of the $\mathbf{R}^{4}$ plane. The fundamental string stretched 
between the D4-D4' system of section 2 now becomes a D2-brane stretched between the $\mathrm{D} 4{ }^{\prime}$ branes and the NS5 branes. From the point of view of the world-volume theory of NS5 branes, this corresponds to the creation of heavy probe strings whose presence leads to the Wilson surfaces in the $6 \mathrm{~d}(2,0)$ theory. The representation carried by these Wilson surfaces can be understood in the following way: to begin with note that there can at most be a single D2-brane stretched between an NS5-brane and a D4'-brane. This is because such a D2-brane is fermionic in nature and therefore this system is governed by the S-rule of Hanany and Witten [40]. Now, a single D2-brane ending on the stack of NS5-branes creates a Wilson surface in the fundamental representation. When there are $m$ D2-branes stretched from a single D4'-brane to the stack of NS5 branes, the fermionic nature of D2branes implies that the Wilson surface is in the rank- $m$ anti-symmetric representation. As a consequence of S-rule, $m$ is constrained to be $m \leq N$.

The above description can now be used to obtain the $2 \mathrm{~d}$ quivers describing the effect of Wilson-surface insertion into the elliptic genera of $6 \mathrm{~d}$ self-dual strings. Once again, let us begin by describing the fundamental Wilson surface. It will be convenient to label the NS5 branes from 1 to $N$ with the order of numbering being from left to right. We then introduce a single D4'-brane to the right of this stack and stretch a D2-brane connecting the $N$-th NS5-brane and the D4'-brane. Let us also suspend $k_{i}$ D2-branes between $i$ 'th and $(i+1)$ 'th NS5-branes. We show this brane configuration in figure 4a. The $2 \mathrm{~d}$ quiver obtained from this configuration is expected to engineer the insertion of fundamental Wilson surface into the elliptic genera. As we will momentarily explain, this is a $\prod_{i=1}^{N-1} \mathrm{U}\left(k_{i}\right)$ quiver gauge theory. In the absence of the D4'-brane, this quiver was first described in $[18,19]$. The extra matter introduced into the quiver as a result of including the $\mathrm{D} 4^{\prime}$-branes can be seen by first using Hanany-Witten transition to go to the frame where the D4'-brane lies between the $(N-1)$ 'th and the $N^{\prime}$ 'th NS5-brane (see figure $4 \mathrm{~b}$ ). The D2-brane suspended between the D4'-brane and NS5-brane is annihilated in the process. Open strings stretched between the D4'-brane and the stack of $k_{N-1}$ D2-branes (suspended between the $(N-1)$ 'th and $N$ 'th NS5 branes) will now give rise to a $\mathrm{U}\left(k_{N-1}\right)$-fundamental $(0,4)$ twisted hyper and a $\mathrm{U}\left(k_{N-1}\right)$-fundamental Fermi multiplet transforming as a $\mathrm{SU}(2)_{1 L}$ doublet. Additionally, there are gauge singlet Fermi multiplets coming from the excitations of the open string stretched between the D4'-brane and the D6-brane. The resulting quiver is shown in figure 4c.

Similarly, we can engineer the Wilson surface in the rank- $m$ anti-symmetric irreducible representation by starting with a D4'-brane lying to the right of the NS5-brane stack and stretching $m$ D2-branes from the $\mathrm{D}^{\prime}$ to the NS5-brane stack such that the $i$ 'th D2-brane ends on the $(N+1-i)^{\prime}$ th NS5 brane, for all $1 \leq i \leq m$. This set-up can be dualised by the Hanany-Witten transition, to a frame where the $\mathrm{D} 4^{\prime}$-brane lies between the $(N-m)^{\prime}$ 'th and $(N+1-m)^{\prime}$ 'th NS5-brane with no D2-branes suspended between the D4' and NS5-branes. Open strings stretched between the $\mathrm{D} 4^{\prime}$ and the $k_{N-m}$ D2-branes (suspended between the $(N-m)$ 'th and $(N+1-m)$ 'th NS5 branes) will then give rise to a $\mathrm{U}\left(k_{N-m}\right)$-fundamental twisted hyper and a $\mathrm{U}\left(k_{N-m}\right)$-fundamental Fermi multiplet in $\mathrm{SU}(2)_{1 L}$ doublet. Also, the open strings stretched between the D4'-brane and the D6-brane give rise to a Fermi multiplet transforming as the bifundamental of $\mathrm{U}(1)_{N-m} \times \mathrm{U}(1)$, where the $\mathrm{U}(1)_{N-m}$ factor 


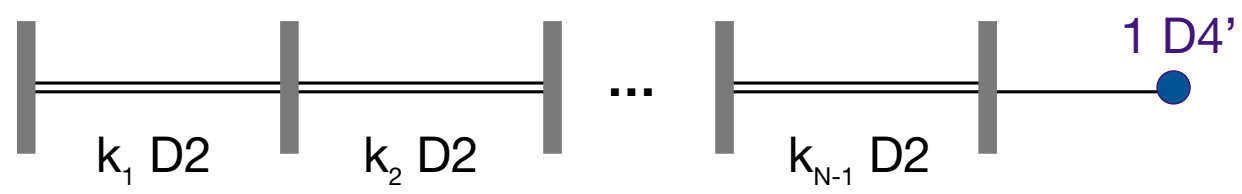

(a) The brane configuration engineering the fundamental Wilson surface of the $6 \mathrm{~d}(2,0)$ theory of type $A_{N-1}$. The vertical lines in the picture denote NS5-branes.

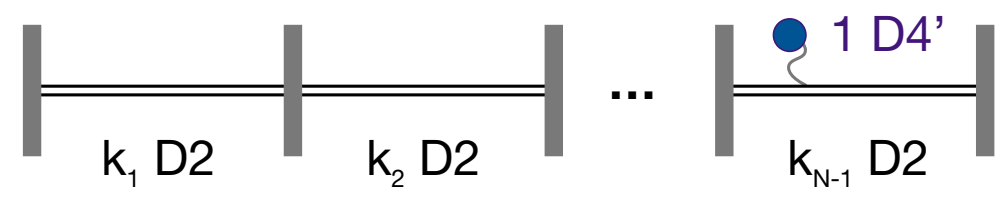

(b) The brane configuration obtained from application of Hanany-Witten transition to the set-up of figure 4a. The vertical lines in the picture denote NS5-branes.

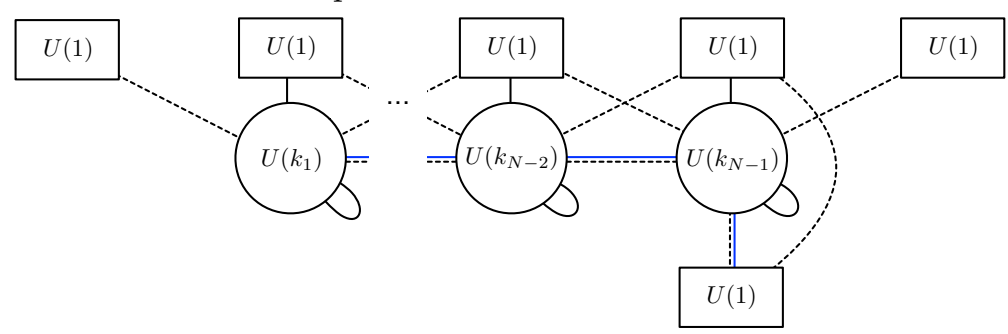

(c) The $2 \mathrm{~d}(0,4)$ quiver gauge theory obtained from the brane configuration of figure $4 \mathrm{~b}$. Here solid black lines denote a hypermultiplet, solid blue lines denote a twisted hyper-multiplet, while the dashed lines denote a Fermi multiplet.

Figure 4. The brane configuration and $2 \mathrm{~d}$ theory describing the fundamental Wilson surface defect. Table 3 also has a D6-brane which has not been shown here.

comes from the $\mathrm{U}(1)$ symmetry associated to the D6 brane segment between the $(N-m)^{\text {'th }}$ and the $(N+1-m)$ 'th NS5-brane and the other $\mathrm{U}(1)$ factor comes from the symmetry associated to the $\mathrm{D} 4$ '-brane. The inclusion of these fields in the $2 \mathrm{~d}$ quiver will therefore capture the effect of a rank- $m$ antisymmetric Wilson surface. We show the relevant brane configuration and $2 \mathrm{~d}$ quiver for the case of a rank- 2 antisymmetric Wilson surface in figure 5 .

In the general case of $N^{\prime}>1$, we can consider brane configurations with $n_{i} \mathrm{D} 4^{\prime}$-branes between the $i$ 'th and the $(i+1)$ 'th NS5 brane, such that $\sum_{i=1}^{N-1} n_{i}=N^{\prime}$ (see figure 6a); from the above discussion it is clear that such configuration will correspond to inserting a Wilson surface in the representation $\mathbf{R}=\otimes_{i=1}^{N-1}\left(\Lambda^{N-i}\right)^{n_{i}}$. The resulting $2 \mathrm{~d}$ quiver is shown in figure $6 \mathrm{~b}$.

Let us now give a more detailed description of the $2 \mathrm{~d}$ gauge theories obtained from the world-volume of D2-branes suspended between NS5-branes along with the insertion of D4'-branes. We consider a stack of $k_{i}$ D2-branes and $n_{i}$ D4'-branes, located in the $x^{9}$ interval between $i$ 'th and $(i+1)$ 'th NS5-branes, for all $i=1, \cdots, N-1$. We draw the given configuration in figure $6 \mathrm{a}$. The chain of $\left(k_{1}, k_{2}, \cdots, k_{N-1}\right) \mathrm{D} 2$-branes engineers a $2 \mathrm{~d}$ linear quiver theory with $\prod_{i=1}^{N-1} \mathrm{U}\left(k_{i}\right)$ gauge symmetry. It inherits $\mathrm{SU}(2)_{1 L} \times \mathrm{SU}(2)_{1 R} \times \mathrm{SU}(2)_{2 R}$ global symmetry of the brane system, where $\mathrm{SU}(2)_{1 R} \times \mathrm{SU}(2)_{2 R}$ is the R-symmetry of $2 \mathrm{~d}$ 


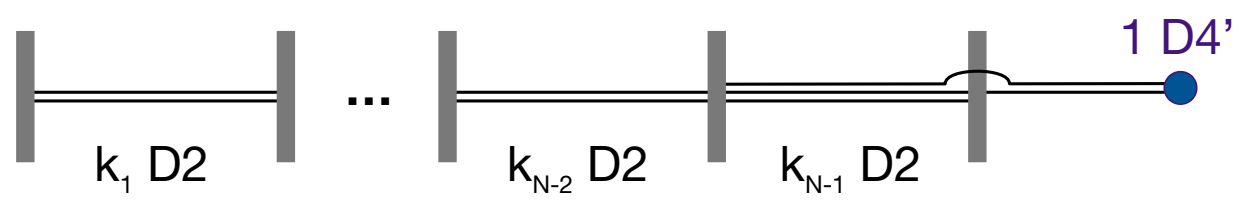

(a) The brane configuration engineering the rank-2 antisymmetric Wilson surface of the $6 \mathrm{~d}(2,0)$ theory of type $A_{N-1}$. The vertical lines in the picture denote NS5-branes.

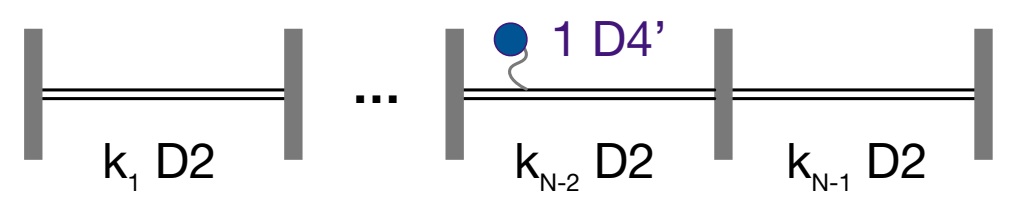

(b) The brane configuration obtained from application of Hanany-Witten transition to the set-up of figure 5a. The vertical lines in the picture denote NS5-branes.

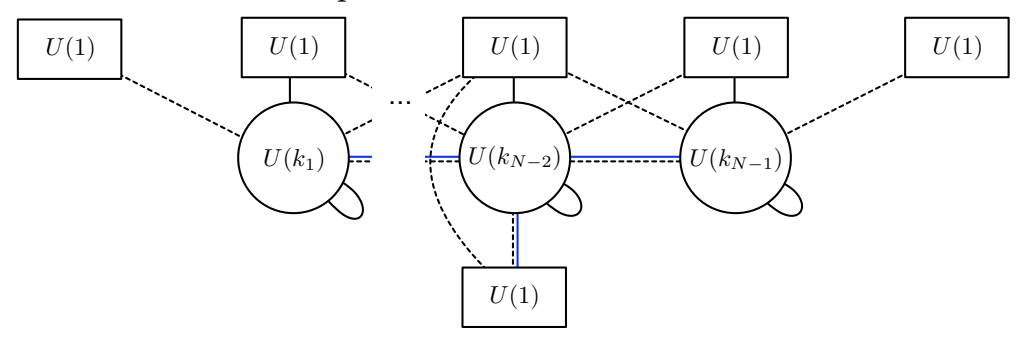

(c) The 2d $(0,4)$ quiver gauge theory obtained from the brane configuration of figure 5b. Here solid black lines denote a hypermultiplet, solid blue lines denote a twisted hyper-multiplet, while the dashed lines denote a Fermi multiplet.

Figure 5. The brane configuration and $2 \mathrm{~d}$ theory describing the rank-2 antisymmetric Wilson surface defect. Table 3 also has a D6-brane which has not been shown here.

$\mathcal{N}=(0,4)$ supersymmetry. At a classical level, it also captures $\prod_{i=1}^{N-1} \mathrm{U}\left(n_{i}\right) \times \prod_{j=0}^{N} \mathrm{U}(1)_{j}$ flavor symmetry of $\left(n_{1}, n_{2}, \cdots, n_{N-1}\right)$ D4'-branes and chain of $(N+1)$ D6-brane segments. Massless excitations coming from open strings ending on D2-branes induce the field content of table 4 , in which the $\mathrm{U}(1)_{i}$ charge of an individual field is denoted by $\mathcal{Q}_{i}$. Only a subset of global U(1)'s will be consistent at a quantum level, yielding no mixed anomaly with gauge symmetry. If one uses $T_{i}, F_{i}, G_{i}$ to denote the Abelian generators of $\mathrm{U}(1) \subset \mathrm{U}\left(k_{i}\right)$, $\mathrm{U}\left(n_{i}\right), \mathrm{U}(1)_{i}$, respectively, then all the non-vanishing mixed anomalies between gauge and global symmetries are

$\operatorname{Tr}\left(\gamma_{3} T_{i} G_{i-1}\right)=-1, \quad \operatorname{Tr}\left(\gamma_{3} T_{i} G_{i}\right)=2, \quad \operatorname{Tr}\left(\gamma_{3} T_{i} G_{i+1}\right)=-1 \quad$ for all $\quad 1 \leq i \leq N-1$

This implies that only two $\mathrm{U}(1)$ 's, out of $\prod_{j=0}^{N} \mathrm{U}(1)_{j}$ global symmetry, will remain at a quantum level. These two U(1)'s are the ones generated by $\sum_{j=0}^{N} G_{j}$ and $\sum_{j=0}^{N}(j-1) G_{j}$. We also notice that the sum of all $\mathrm{U}(1)$ generators $\sum_{j=0}^{N} G_{j}+\sum_{i=1}^{N-1}\left(T_{i}+F_{i}\right)$ trivially acts on the fields, therefore $\sum_{j=0}^{N} G_{j} \simeq-\sum_{i=1}^{N-1}\left(T_{i}+F_{i}\right)$ effectively.

One can easily construct the Lagrangian of the quiver gauge theory. We will use the $\mathcal{N}=(0,2)$ SUSY formalism [41] and write the holomorphic potentials $E_{\eta}$ and $J_{\eta}$, for each 


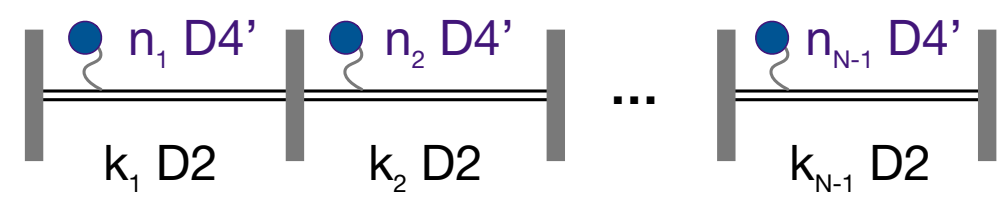

(a) The brane configuration for the Wilson surface in the representation $\mathbf{R}=\otimes_{i=1}^{N-1}\left(\bigwedge^{N-i}\right)^{n_{i}}$. The vertical lines in the picture denote NS5-branes.

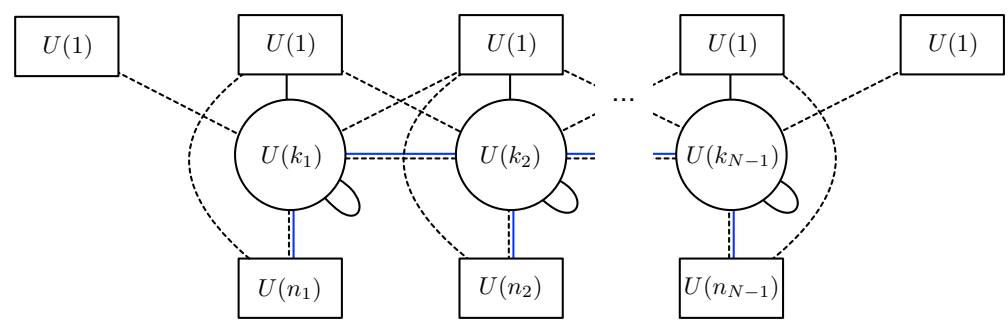

(b) The 2d $(0,4)$ quiver gauge theory obtained from the brane configuration of figure 6a. Here solid black lines denote a hypermultiplet, solid blue lines denote a twisted hyper-multiplet, while the dashed lines denote a Fermi multiplet.

Figure 6. The brane configuration and $2 \mathrm{~d}$ theory describing a Wilson surface defect in the representation $\mathbf{R}=\otimes_{i=1}^{N-1}\left(\bigwedge^{N-i}\right)^{n_{i}}$. Table 3 also has a D6-brane which has not been shown here.

$(0,2)$ Fermi multiplet $\eta$, in a way which ensures the $\mathcal{N}=(0,4)$ SUSY enhancement [24]. First, we decompose the $(0,4)$ vector and (twisted) hypermultiplets into the $(0,2)$ chiral and Fermi multiplets as follows:

$$
\begin{array}{r}
\operatorname{vector}\left(A_{\mu}^{(i)}, \zeta_{\dot{\alpha}}^{(i) A}\right) \longrightarrow \operatorname{vector} V^{(i)}\left(A_{\mu}^{(i)}, \zeta_{\dot{1}}^{(i) 1}\right) \oplus \operatorname{Fermi} \Lambda^{(i)}\left(\zeta_{\dot{2}}^{(i) 1}\right) \\
\text { hyper }\left(a_{\alpha \dot{\alpha}}^{(i)}, \lambda_{\alpha}^{(i) A}\right) \longrightarrow \operatorname{chiral} B^{(i)}\left(a_{1 \dot{1}}^{(i)}, \lambda_{1}^{(i) 2}\right) \oplus \operatorname{chiral} \tilde{B}^{(i) \dagger}\left(a_{1 \dot{2}}^{(i)}, \lambda_{1}^{(i) 1}\right) \\
\text { hyper }\left(q_{\dot{\alpha}}^{(i)}, \psi^{(i) A}\right) \longrightarrow \operatorname{chiral} q^{(i)}\left(q_{\dot{1}}^{(i)}, \psi^{(i) 2}\right) \oplus \operatorname{chiral} \tilde{q}^{(i) \dagger}\left(q_{\dot{2}}^{(i)}, \psi^{(i) 1}\right) \\
\text { twisted hyper }\left(\Phi_{A}^{(i)}, \Psi_{\dot{\alpha}}^{(i)}\right) \longrightarrow \operatorname{chiral} \Phi^{(i)}\left(\Phi_{1}^{(i)}, \Psi_{\dot{1}}^{(i)}\right) \oplus \operatorname{chiral} \tilde{\Phi}^{(i) \dagger}\left(\Phi_{2}^{(i)}, \Psi_{\dot{2}}^{(i)}\right) \\
\text { twisted hyper }\left(\varphi_{A}^{(i)}, \xi_{\dot{\alpha}}^{(i)}\right) \longrightarrow \operatorname{chiral} \varphi^{(i)}\left(\varphi_{1}^{(i)}, \xi_{\dot{1}}^{(i)}\right) \oplus \operatorname{chiral} \tilde{\varphi}^{(i) \dagger}\left(\varphi_{2}^{(i)}, \xi_{\dot{2}}^{(i)}\right) .
\end{array}
$$

On the other hand, all $(0,4)$ Fermi multiplets $\Psi_{\alpha}^{(i)}, \chi^{(i)}, \bar{\chi}^{(i)}, \xi_{\alpha}^{(i)}, \rho^{(i)}$ are also $(0,2)$ Fermi multiplets. Second, we introduce $E_{\Lambda}^{(i)}$ and $J_{\Lambda}^{(i)}$ for Fermi multiplets $\Lambda^{(i)}$ as functions of $(0,2)$ chiral superfields. We follow [24] which introduces the chiral multiplets from (twisted) hypermultiplets to $J_{\Lambda}^{(i)}\left(E_{\Lambda}^{(i)}\right)$, i.e.,

$$
J_{\Lambda}^{(i)}=q^{(i)} \tilde{q}^{(i)}+\left[B^{(i)}, \tilde{B}^{(i)}\right], \quad E_{\Lambda}^{(i)}=\varphi^{(i)} \tilde{\varphi}^{(i)}-\tilde{\varphi}^{(i-1)} \varphi^{(i-1)}+\Phi^{(i)} \tilde{\Phi}^{(i)}
$$

with $\varphi^{(0)}, \tilde{\varphi}^{(0)}, \varphi^{(N)}, \tilde{\varphi}^{(N)}$ understood as being null. Third, the above $J_{\Lambda}^{(i)}$ and $E_{\Lambda}^{(i)}$ must be accompanied by other holomorphic potentials to satisfy the $(0,2)$ SUSY preserving condition $\sum_{\eta} \operatorname{Tr}\left(J_{\eta} \cdot E_{\eta}\right)=0$, where the sum is taken over all Fermi multiplets in the 


\begin{tabular}{|c|c|ccc|}
\hline Type & Fields & $\mathrm{U}\left(k_{i}\right)$ & $\mathrm{U}\left(n_{i}\right)$ & $\left(\mathcal{Q}_{i-1}, \mathcal{Q}_{i}, \mathcal{Q}_{i+1}\right)$ \\
\hline vector & $A_{\mu}^{(i)}, \zeta_{\dot{\alpha}}^{(i) A}$ & $\mathbf{a d j}$ & $\mathbf{1}$ & $(0,0,0)$ \\
hyper & $a_{\alpha \dot{\alpha}}^{(i)}, \lambda_{\alpha}^{(i) A}$ & $\mathbf{a d j}$ & $\mathbf{1}$ & $(0,0,0)$ \\
twisted & $\Phi_{A}^{(i)}, \Psi_{\dot{\alpha}}^{(i)}$ & $\mathbf{k}_{i}$ & $\overline{\mathbf{n}}_{i}$ & $(0,0,0)$ \\
Fermi & $\Psi_{\alpha}^{(i)}$ & $\mathbf{k}_{i}$ & $\overline{\mathbf{n}}_{i}$ & $(0,0,0)$ \\
hyper & $q_{\dot{\alpha}}^{(i)}, \psi^{(i) A}$ & $\mathbf{k}_{i}$ & $\mathbf{1}$ & $(0,+1,0)$ \\
Fermi & $\chi^{(i)}$ & $\mathbf{k}_{i}$ & $\mathbf{1}$ & $(0,0,-1)$ \\
Fermi & $\bar{\chi}^{(i)}$ & $\overline{\mathbf{k}}_{i}$ & $\mathbf{1}$ & $(+1,0,0)$ \\
\hline
\end{tabular}

\begin{tabular}{|c|c|cc|}
\hline Type & Fields & $\mathrm{U}\left(k_{i}\right)$ & $\mathrm{U}\left(k_{i+1}\right)$ \\
\hline twisted & $\varphi_{A}^{(i)}, \xi_{\dot{\alpha}}^{(i)}$ & $\mathbf{k}_{i}$ & $\overline{\mathbf{k}}_{i+1}$ \\
Fermi & $\xi_{\alpha}^{(i)}$ & $\mathbf{k}_{i}$ & $\overline{\mathbf{k}}_{i+1}$ \\
\hline
\end{tabular}

(b) between $i$ 'th and $(i+1)^{\prime}$ th nodes $(i \leq$ $N-2)$.

\begin{tabular}{|c|c|cc|}
\hline Type & Fields & $\mathrm{U}\left(n_{i}\right)$ & $\mathcal{Q}_{i}$ \\
\hline Fermi & $\rho^{(i)}$ & $\mathbf{n}_{i}$ & -1 \\
\hline
\end{tabular}

(a) in the $i$ 'th gauge node $(i \leq N-1)$.

(c) between $i^{\prime}$ th D6- and D4-branes $(i \leq$ $N-1)$.

Table 4. $\mathcal{N}=(0,4)$ multiplets in the quiver gauge theory. 'twisted' means a twisted hypermultiplet.

theory. Allowed are the following potentials:

$$
\begin{aligned}
& E_{\chi}^{(i)}=\varphi^{(i)} q^{(i+1)}, \quad J_{\chi}^{(i)}=\tilde{q}^{(i+1)} \tilde{\varphi}^{(i)}, \\
& E_{\bar{\chi}}^{(i)}=\tilde{q}^{(i-1)} \varphi^{(i-1)}, \quad J_{\bar{\chi}}^{(i)}=-\tilde{\varphi}^{(i-1)} q^{(i-1)}, \\
& E_{\Psi_{1}}^{(i)}=B^{(i)} \Phi^{(i)}, \quad J_{\Psi_{1}}^{(i)}=\tilde{\Phi}^{(i)} \tilde{B}^{(i)}, \quad E_{\Psi_{2}}^{(i)}=-\tilde{B}^{(i)} \Phi^{(i)}, \quad J_{\Psi_{2}}^{(i)}=\tilde{\Phi}^{(i)} B^{(i)}, \\
& E_{\xi_{1}}^{(i)}=\varphi^{(i)} B^{(i+1)}-B^{(i)} \varphi^{(i)}, \quad E_{\xi_{2}}^{(i)}=\tilde{B}^{(i)} \varphi^{(i)}-\varphi^{(i)} \tilde{B}^{(i+1)}, \quad E_{\rho}^{(i)}=\tilde{\Phi}^{(i)} q^{(i)}, \\
& J_{\xi_{1}}^{(i)}=\tilde{B}^{(i+1)} \tilde{\varphi}^{(i)}-\tilde{\varphi}^{(i)} \tilde{B}^{(i)}, \quad J_{\xi_{2}}^{(i)}=B^{(i+1)} \tilde{\varphi}^{(i)}-\tilde{\varphi}^{(i)} B^{(i)}, \quad J_{\rho}^{(i)}=-\tilde{q}^{(i)} \Phi^{(i)} .
\end{aligned}
$$

With the following D-term potential $D^{(i)}$,

$$
\begin{aligned}
D^{(i)}= & q^{(i)} q^{(i) \dagger}-\tilde{q}^{(i) \dagger} \tilde{q}^{(i)}+\left[B^{(i)}, B^{(i) \dagger}\right]-\left[\tilde{B}^{(i) \dagger}, \tilde{B}^{(i)}\right] \\
& +\Phi^{(i)} \Phi^{(i) \dagger}-\tilde{\Phi}^{(i) \dagger} \tilde{\Phi}^{(i)}-\varphi^{(i-1) \dagger} \varphi^{(i-1)}+\tilde{\varphi}^{(i-1)} \tilde{\varphi}^{(i-1) \dagger}+\varphi^{(i)} \varphi^{(i) \dagger}-\tilde{\varphi}^{(i) \dagger} \tilde{\varphi}^{(i)},
\end{aligned}
$$

the bosonic potential $V=\sum_{\eta}\left(\left|E_{\eta}\right|^{2}+\left|J_{\eta}\right|^{2}\right)+\frac{1}{4} \sum_{i=1}^{N-1}\left(D^{(i)}\right)^{2}$ can be arranged into

$$
\begin{aligned}
V= & \sum_{i=1}^{N-1}\left(\frac{1}{4}\left(\left(\sigma^{m}\right)^{\dot{\alpha}} q_{\dot{\alpha}}^{(i)} q^{(i) \dagger \dot{\beta}}+\frac{1}{2}\left(\sigma^{m}\right)^{\dot{\alpha}} \dot{\beta}\left[a_{\alpha \dot{\alpha}}^{(i)}, a^{(i) \alpha \dot{\beta}}\right]\right)^{2}\right. \\
& +\frac{1}{4}\left(\left(\sigma^{I}\right)^{A}{ }_{B} \varphi_{A}^{(i)} \varphi^{(i) \dagger B}-\left(\sigma^{I}\right)^{A}{ }_{B} \varphi^{(i-1) \dagger B} \varphi_{A}^{(i-1)}+\left(\sigma^{I}\right)^{A}{ }_{B} \Phi_{A}^{(i)} \Phi^{(i) \dagger B}\right)^{2} \\
& \left.+\frac{1}{2}\left|q_{\dot{\alpha}}^{(i)} \Phi_{A}^{(i)}\right|^{2}+\frac{1}{2}\left|q_{\dot{\alpha}}^{(i)} \varphi_{A}^{(i-1) \dagger}\right|+\frac{1}{2}\left|q_{\dot{\alpha}}^{(i)} \varphi_{A}^{(i)}\right|+\frac{1}{2}\left|a_{\alpha \dot{\alpha}}^{(i)} \Phi_{A}^{(i)}\right|^{2}+\frac{1}{2}\left|\varphi_{A}^{(i)} a_{\alpha \dot{\alpha}}^{(i+1)}-a_{\alpha \dot{\alpha}}^{(i)} \varphi_{A}^{(i)}\right|^{2}\right) .
\end{aligned}
$$

Note that though we necessarily need the Fermi fields $\rho$ in order to write a Lagrangian with $(0,4)$ SUSY, we expect that these will decouple from the interacting system in the IR. A simple way to see this is to notice that they are gauge invariant chiral operators and hence their IR scaling dimensions can be deduced from their charge with respect to the R-symmetry of $(0,2)$ subalgebra. The R-current of $(0,2)$ subalgebra will be given by a linear combination of the two Cartans of the $\mathrm{SO}(4)_{R}$-symmetry acting on the UV $(0,4)$ 
algebra. However, $\rho$ is a singlet of this $\mathrm{SO}(4)_{R}$ symmetry and hence it will carry a zero charge with respect to the $(0,2) \mathrm{R}$-symmetry. Since the right moving dimension of chiral operators is proportional to their R-charge, it implies that for $\rho, \bar{L}_{0}=0$. Their left moving dimension $L_{0}$ can also be fixed by using the fact that $L_{0}-\bar{L}_{0}=\frac{1}{2}$, since the difference of the left and right moving dimensions is given by the spin of the operators. Thus we find that the fermions $\rho$ must have $L_{0}=\frac{1}{2}$ and $\bar{L}_{0}=0$. This is the unitarity bound for left moving gauge invariant fermions to become free, much like in $[42,43]$.

\subsection{Elliptic genera with Wilson surfaces}

We consider the elliptic genera of $2 \mathrm{~d} \mathcal{N}=(0,4)$ quiver gauge theories, introduced in section 3.1. They are labeled by the ranks of the $N-1$ product gauge and flavor groups $\left\{k_{1}, k_{2}, \cdots, k_{N-1} \mid n_{1}, n_{2}, \cdots, n_{N-1}\right\}$, where $\left\{k_{1}, k_{2}, \cdots, k_{N-1}\right\}$ and $\left\{n_{1}, n_{2}, \cdots, n_{N-1}\right\}$ correspond to the winding numbers for different species of self-dual strings and defect strings. One can express them in the following trace representation:

$$
\mathcal{I}_{\left\{k_{1}, \cdots, k_{N-1}\right\}}^{\left\{n_{1}, \cdots, n_{N-1}\right\}}=\operatorname{Tr}_{R R}\left[(-1)^{F} q^{H_{L}} \bar{q}^{H_{R}} t^{2\left(J_{r}-J_{R}\right)} u^{2 J_{l}} \mu^{\sum_{j=0}^{N}(j-1) G_{j}} \prod_{i=1}^{N-1} \prod_{\ell=1}^{n_{i}} e^{-m_{\ell}^{(i)} Q_{\ell}^{(i)}}\right]
$$

The elliptic genus captures the BPS states annihilated by $Q^{1 \dot{1}}$ and $Q^{2 \dot{2}}$, satisfying $H_{R} \sim$ $\left\{Q^{1 \dot{1}}, Q^{2 \dot{2}}\right\}$. Most of the fugacity variables and conjugate charges were introduced in section 2.2. In addition, $m_{\ell}^{(i)}$ are chemical potentials for $\mathrm{U}\left(n_{i}\right)$ flavor symmetry, whose Cartan generators are denoted as $Q_{\ell}^{(i)}$. All these deformations made by chemical potentials are crucial for regulating the infrared divergences.

Evaluation of the elliptic genus of $2 \mathrm{~d} \mathcal{N}=(0,2)$ gauge theory was formulated in [44-46] by means of supersymmetric localization. Saddle points in the path integral are characterized by the gauge flat connections on $T^{2}$. We denote eigenvalues of the $\mathrm{U}\left(k_{i}\right)$ gauge connection by $\phi^{(i)}=\left\{\phi_{1}^{(i)}, \phi_{2}^{(i)}, \cdots, \phi_{k_{i}}^{(i)}\right\}$, and the collection of all gauge connections by $\phi=\left\{\phi^{(1)}, \ldots, \phi^{(N-1)}\right\}$. All the Gaussian fluctuations around saddle points can be integrated out, leaving us with the integration over zero modes of the 1-loop determinant:

$\mathcal{I}_{\left\{k_{1}, \cdots, k_{N-1}\right\}}^{\left\{n_{1}, \cdots, n_{N-1}\right\}}=\frac{1}{\prod_{i=1}^{N-1} k_{i} !} \oint\left[\prod_{i=1}^{N-1} \prod_{s=1}^{k_{i}} \frac{d \phi_{s}^{(i)}}{2 \pi i}\right] Z_{k_{1} \ldots k_{N-1}}^{\mathrm{NS} 5}\left(\phi, \epsilon_{1,2}\right) Z_{k_{1} \ldots k_{N-1}}^{\mathrm{D} 6}\left(\phi, m, \epsilon_{1,2}\right) \prod_{i=1}^{N-1} \prod_{\ell=1}^{n_{i}} Z_{k_{i}}^{\mathrm{D} 4}\left(\phi^{(i)}, m_{\ell}^{(i)}, \epsilon_{1,2}\right)$,

where $\left(\right.$ for $\left.\theta(z)=\theta_{1}\left(\tau, \frac{z}{2 \pi i}\right)\right)$

$$
\begin{aligned}
Z_{k_{1} \ldots k_{N-1}}^{\mathrm{NS} 5}\left(\phi, \epsilon_{1,2}\right)= & \prod_{i=1}^{N-1}\left(\frac{\eta^{3} \theta\left(2 \epsilon_{+}\right)}{\theta\left(\epsilon_{1}\right) \theta\left(\epsilon_{2}\right)}\right)^{k_{i}} \prod_{i=1}^{N-1} \prod_{s \neq t}^{k_{i}} \frac{\theta\left(\phi_{s}^{(i)}-\phi_{t}^{(i)}\right) \theta\left(\phi_{s}^{(i)}-\phi_{t}^{(i)}+2 \epsilon_{+}\right)}{\theta\left(\phi_{s}^{(i)}-\phi_{t}^{(i)}+\epsilon_{1}\right) \theta\left(\phi_{s}^{(i)}-\phi_{t}^{(i)}+\epsilon_{2}\right)} \\
& \prod_{i=1}^{N-1} \prod_{s=1}^{k_{i}} \prod_{t=1}^{k_{i+1}} \frac{\theta\left(\phi_{t}^{(i+1)}-\phi_{s}^{(i)}+\epsilon_{-}\right) \theta\left(-\phi_{t}^{(i+1)}+\phi_{s}^{(i)}+\epsilon_{-}\right)}{\theta\left(\phi_{t}^{(i+1)}-\phi_{s}^{(i)}-\epsilon_{+}\right) \theta\left(-\phi_{t}^{(i+1)}+\phi_{s}^{(i)}-\epsilon_{+}\right)}
\end{aligned}
$$




$$
\begin{aligned}
Z_{k_{1} \ldots k_{N-1}}^{\mathrm{D} 6}\left(\phi, m, \epsilon_{1,2}\right) & =\prod_{i=1}^{N-1} \prod_{s=1}^{k_{i}} \frac{\theta\left(m+(1-i) m+\phi_{s}^{(i)}\right) \theta\left(m-(1-i) m-\phi_{s}^{(i)}\right)}{\theta\left(\epsilon_{+}+(1-i) m+\phi_{s}^{(i)}\right) \theta\left(\epsilon_{+}-(1-i) m-\phi_{s}^{(i)}\right)}, \\
Z_{k_{i}}^{\mathrm{D} 4}\left(\phi^{(i)}, m_{\ell}^{(i)}, \epsilon_{1,2}\right) & =\frac{\theta\left(m_{\ell}^{(i)}-i m\right)}{\eta} \prod_{s=1}^{k_{i}} \frac{\theta\left(\phi_{s}^{(i)}-m_{\ell}^{(i)}+\epsilon_{-}\right) \theta\left(-\phi_{s}^{(i)}+m_{\ell}^{(i)}+\epsilon_{-}\right)}{\theta\left(\phi_{s}^{(i)}-m_{\ell}^{(i)}-\epsilon_{+}\right) \theta\left(-\phi_{s}^{(i)}+m_{\ell}^{(i)}-\epsilon_{+}\right)} .
\end{aligned}
$$

The next step is to integrate over all saddle points, parametrized by eigenvalues $\phi$ of the $\prod_{i=1}^{N-1} \mathrm{U}\left(k_{i}\right)$ gauge connection. This zero-mode integral becomes a contour integral over the space of eigenvalues, whose integral contour is specified by the Jeffrey-Kirwan residue operation $[45,46]$. The selection of individual residue depends on an auxiliary vector $\eta$, while the final residue sum is independent of it.

It is known that in the special case of $n_{1}=\cdots=n_{N-1}=0$, the Jeffrey-Kirwan residues are classified by $(N-1)$-tuples of Young tableaux [47, 48] with the choice of $\eta=(1,1, \cdots, 1)$. Each residue corresponds to a possible configuration of the Young tableaux $\left(Y_{1}, \cdots, Y_{N-1}\right)$ with $\left|Y_{i}\right|=k_{i}$. A box $s=(m, n)$ in the $i$ 'th Young tableau $Y_{i}$ represents the following pole location for an integral variable $\phi^{(i)}$ :

$$
\phi^{(i)}=(i-1) m-\epsilon_{+}-(n-1) \epsilon_{1}-(m-1) \epsilon_{2} .
$$

The classification of JK residues becomes more complicated after introducing the $\prod_{i=1}^{N-1} \mathrm{U}\left(n_{i}\right)$ flavor symmetry of $\mathrm{D} 4^{\prime}$-branes. We must include new types of residues, being associated to the poles at

$$
\phi^{(i)}=\epsilon_{+}+m_{\ell=1, \cdots n_{i}}^{(i)} \quad \text { and } \quad \phi^{(i \pm 1)}-\phi^{(i)}=\epsilon_{+}
$$

in addition to the original ones (3.10). For example, with $N=2$, all residues are labeled by the Young tableau $Y_{1}$ and the $n_{1}$-dimensional vector $\mathbf{u}$ such that $\left|Y_{1}\right|=k_{1}-|\mathbf{u}|$, where each component of the vector $\mathbf{u}$ must be either 0 or 1 . $|\mathbf{u}|$ denotes the sum of all components of the vector $\mathbf{u}$. A non-zero, $\nu^{\prime}$ th component of $\mathbf{u}$ encodes an integral variable $\phi^{(1)}$ to take the following value:

$$
\phi^{(1)}=\epsilon_{+}+m_{\nu}^{(1)}
$$

The associated residues are obtained by substituting these values to the integral measure (3.8). Similarly for general $N$, one obtains the elliptic genus $\mathcal{I}_{\left\{k_{1}, \cdots, k_{N-1}\right\}}^{\left\{n_{1}, \cdots, n_{N-1}\right\}}$ of self-dual strings by summing over all possible JK residues associated to (3.10) and (3.11).

\subsection{Relation to 5d Wilson loops}

As the individual elliptic genus represents the BPS states for a given configuration of selfdual strings, the full $6 \mathrm{~d}$ partition function must be written as their sum. We note that for a given configuration $\left(n_{1}, \cdots, n_{N-1}\right)$ of $\mathrm{D} 4^{\prime}$-branes, all the elliptic genera with different winding numbers share a common factor of $Z_{\mathrm{D} 6-\mathrm{D} 4^{\prime}}^{\left\{n_{1}, \cdots, n_{N-1}\right\}}=\prod_{i=1}^{N-1} \prod_{\ell=1}^{n_{i}} \theta\left(m_{\ell}^{(i)}-i m\right) / \eta$ which is the 1-loop determinant contribution of D6-D4 $4^{\prime}$ bifundamental Fermi multiplets. As 
explained at the end of section 3.1, the D6-D4' fermions are decoupled from the interacting IR dynamics. Therefore, we divide the elliptic genera $\mathcal{I}_{\left\{k_{1}, \cdots, k_{N-1}\right\}}^{\left\{n_{1}, \cdots, n_{N-1}\right\}}$ by $Z_{\mathrm{D} 6-\mathrm{D} 4^{\prime}}^{\left\{n_{1}, \cdots, n_{N-1}\right\}}$. The grand canonical partition is then given by

$$
Z_{6 d / 2 d}^{\left\{n_{1}, \cdots, n_{N-1}\right\}}=\mathcal{I}_{0}^{N} \cdot\left(\sum_{k_{1}, \ldots, k_{N-1} \geqslant 0} \mathfrak{n}_{1}^{k_{1}} \mathfrak{n}_{2}^{k_{2}} \cdots \mathfrak{n}_{N-1}^{k_{N-1}} \cdot \mathcal{I}_{\left\{k_{1}, \cdots, k_{N-1}\right\}}^{\left\{n_{1}, \cdots, n_{N-1}\right\}} / Z_{\mathrm{D} 6-\mathrm{D} 4^{\prime}}^{\left\{n_{1}, \cdots, n_{N-1}\right\}}\right)
$$

$\mathfrak{n}_{1, \ldots, N-1}$ denote the winding number fugacities for $(N-1)$ different types of self-dual strings. The overall multiplicative factor $\mathcal{I}_{0}^{N}$ captures pure momentum states that are decoupled from stringy modes at low energy. It takes the following form:

$$
\mathcal{I}_{0}^{N}=\mathrm{PE}\left[N \frac{\operatorname{sh}\left(m \pm \epsilon_{-}\right)}{\operatorname{sh}\left(\epsilon_{+} \pm \epsilon_{-}\right)} \frac{q}{1-q}\right]=\operatorname{PE}\left[N \frac{t\left(\mu+\mu^{-1}-u-u^{-1}\right)}{(1-t u)\left(1-t u^{-1}\right)} \frac{q}{1-q}\right] .
$$

$Z_{6 d / 2 d}^{\left\{n_{1}, \cdots, n_{N-1}\right\}}$ is expected to be the Wilson surface operator in M5-branes, whose representation is determined by the arrangement of $\mathrm{D} 4$ '-branes, i.e., $\left(n_{1}, n_{2}, \cdots, n_{N-1}\right)$, as explained in section 3.1. We will see that this expectation becomes true for minuscule representations, while there will be extra subtleties for non-minuscule representations.

We have two different methods of computing the Wilson surface operator in M5-branes. Recall that $5 \mathrm{~d} \mathcal{N}=1^{*} \mathrm{U}(N)$ gauge theory has the UV completion with non-perturbative corrections, as 6d $(2,0)$ SCFT on $S^{1}$ describing a stack of $N$ parallel M5-branes [49-51]. The 5d SYM instantons are the Kaluza-Klein momentum modes along the compactified circle. Similarly, the W-bosons in the Coulomb branch of 5d SYM are uplifted to the $6 \mathrm{~d}$ self-dual strings, whose tension is proportional to the distance between a pair of M5-branes. Once we identify the instanton fugacity and Coulomb branch parameters of 5d SYM with the $6 \mathrm{~d}$ momentum and winding fugacities along $T^{2}$,

$$
q=e^{2 \pi i \tau}, \quad \mathfrak{n}_{1}=\frac{w_{1}}{w_{2}}, \quad \mathfrak{n}_{2}=\frac{w_{2}}{w_{3}}, \quad \cdots, \quad \mathfrak{n}_{N-1}=\frac{w_{N-1}}{w_{N}} \quad\left(\text { with } w_{1} w_{2} \cdots w_{N}=1\right),
$$

the various Wilson lines in $5 \mathrm{~d} \mathcal{N}=1^{*} \mathrm{SYM}$, studied in section 2.3 , are naturally interpreted as the $6 \mathrm{~d}$ Wilson surface observable in M5-branes. More concretely, we propose the following equalities:

$$
Z_{\text {pert }}^{\mathrm{U}(N)} \cdot \mathcal{W}_{\mathbf{R}}^{\mathrm{U}(N)}=\left(\prod_{i=1}^{N-1} \mathfrak{n}_{i}^{\lambda_{i}}\right) \cdot Z_{6 d / 2 d}^{\left\{n_{1}, \cdots, n_{N-1}\right\}} \quad \text { with } \quad \mathbf{R}=\otimes_{i=1}^{N-1}\left(\bigwedge^{N-i}\right)^{n_{i}}
$$

where $\left(\lambda_{1}, \cdots, \lambda_{N-1}\right)$ denotes the lowest weight of the representation $\mathbf{R}$ in $\alpha$-basis. One necessary condition for (3.16) to hold is that the flavor chemical potentials $m_{\ell}^{(i)}$ must disappear from the final expression of the 6d string elliptic genus $\mathcal{I}_{\left\{k_{1}, \cdots, k_{N-1}\right\}}^{\left\{n_{1}, \cdots, n_{N-1}\right\}} / Z_{\mathrm{D} 6-\mathrm{D} 4^{\prime}}^{\left\{n_{1}, \cdots, n_{N-1}\right\}}$. It turns out to be true for the cases with $N^{\prime}=\sum_{i=1}^{N-1} n_{i}=1$, which corresponds to the Wilson surfaces in minuscule representations. For non-minuscule representations, i.e. $N^{\prime}=\sum_{i=1}^{N-1} n_{i}>1$, we will report the prescription for the flavor fugacities $e^{-m_{\ell}^{(i)}}$, under which (3.16) holds true in most of the winding sectors. 
Since the 5 d perturbative index $Z_{\text {pert }}^{\mathrm{U}(N)}$ and the $\mathrm{U}(1)$ factors $\mathcal{I}_{0}^{N}$ are commonly shared among the Wilson surfaces in different representations, we find it convenient to normalize (3.16) by the partition function $Z_{\text {pert }}^{\mathrm{U}(N)} \cdot \mathcal{W}_{\mathbf{1}}^{\mathrm{U}(N)}=Z_{6 d / 2 d}^{\{0, \cdots, 0\}}[18]$. We will test if the following is true:

$$
\left(\prod_{i=1}^{N-1} \mathfrak{n}_{i}^{-\lambda_{i}}\right) \cdot \frac{\mathcal{W}_{\mathbf{R}}^{\mathrm{U}(N)}}{\mathcal{W}_{\mathbf{1}}^{\mathrm{U}(N)}}=\frac{Z_{6 d / 2 d}^{\left\{n_{1}, \cdots, n_{N-1}\right\}}}{Z_{6 d / 2 d}^{\{0, \cdots, 0\}}} \quad \text { with } \quad \mathbf{R}=\otimes_{i=1}^{N-1}\left(\bigwedge^{N-i}\right)^{n_{i}}
$$

Minuscule representations. When $N=2$ and $n_{1}=1$, we found an agreement between the 6d BPS partition function (3.13) and the $\mathrm{U}(2)$ fundamental Wilson loop $\mathcal{W}_{\mathbf{2}}^{\mathrm{U}(2)}$, by taking the double series expansion in the momentum (instanton) fugacity $q$ and the winding (W-boson) fugacity $\mathfrak{n}_{1}=w_{1}^{2}$, i.e.,

$$
\mathfrak{n}_{1}^{1 / 2} \cdot \mathcal{W}_{\mathbf{2}}^{\mathrm{U}(2)} / \mathcal{W}_{\mathbf{1}}^{\mathrm{U}(2)}=Z_{6 d / 2 d}^{\{1\}} / Z_{6 d / 2 d}^{\{0\}}
$$

verified up to $q^{4}$ and $\mathfrak{n}_{1}^{4}$ order. For $N=3$ case, the 6 d observables $Z_{6 d / 2 d}^{\{0,1\}}$ and $Z_{6 d / 2 d}^{\{1,0\}}$ must be compared with the $\mathrm{U}(3)$ Wilson loops in the $\Lambda^{1}=\mathbf{3}$ and $\Lambda^{2}=\overline{\mathbf{3}}$ representations, respectively. We found

$$
\begin{aligned}
& \mathfrak{n}_{1}^{1 / 3} \mathfrak{n}_{2}^{2 / 3} \cdot \mathcal{W}_{\mathbf{3}}^{\mathrm{U}(3)} / \mathcal{W}_{\mathbf{1}}^{\mathrm{U}(3)}=Z_{6 d / 2 d}^{\{0,1\}} / Z_{6 d / 2 d}^{\{0,0\}} \\
& \mathfrak{n}_{1}^{2 / 3} \mathfrak{n}_{2}^{1 / 3} \cdot \mathcal{W}_{\overline{\mathbf{3}}}^{\mathrm{U}(3)} / \mathcal{W}_{\mathbf{1}}^{\mathrm{U}(3)}=Z_{6 d / 2 d}^{\{1,0\}} / Z_{6 d / 2 d}^{\{0,0\}}
\end{aligned}
$$

verified up to $q^{2}$ and $\mathfrak{n}_{1}^{2} \mathfrak{n}_{2}^{2}$ orders. Similarly for $N=4$ case, up to $q^{2}$ and $\mathfrak{n}_{1}^{2} \mathfrak{n}_{2}^{2} \mathfrak{n}_{3}^{2}$ orders, we checked

$$
\begin{gathered}
\mathfrak{n}_{1}^{1 / 4} \mathfrak{n}_{2}^{1 / 2} \mathfrak{n}_{3}^{3 / 4} \cdot \mathcal{W}_{\mathbf{4}}^{\mathrm{U}(4)} / \mathcal{W}_{\mathbf{1}}^{\mathrm{U}(4)}=Z_{6 d / 2 d}^{\{0,0,1\}} / Z_{6 d / 2 d}^{\{0,0,0\}}, \\
\mathfrak{n}_{1}^{3 / 4} \mathfrak{n}_{2}^{1 / 2} \mathfrak{n}_{3}^{1 / 4} \cdot \mathcal{W}_{\overline{\mathbf{4}}}^{\mathrm{U}(4)} / \mathcal{W}_{\mathbf{1}}^{\mathrm{U}(4)}=Z_{6 d / 2 d}^{\{1,0,0\}} / Z_{6 d / 2 d}^{\{0,0,0\}} \\
\mathfrak{n}_{1}^{1 / 2} \mathfrak{n}_{2}^{1} \mathfrak{n}_{3}^{1 / 2} \cdot \mathcal{W}_{\mathbf{6}}^{\mathrm{U}(4)} / \mathcal{W}_{\mathbf{1}}^{\mathrm{U}(4)}=Z_{6 d / 2 d}^{\{0,1,0\}} / Z_{6 d / 2 d}^{\{0,0,0\}}
\end{gathered}
$$

Non-minuscule representations. On the contrary, the flavor chemical potentials $m_{\ell}^{(i)}$ do not generally disappear from the elliptic genera $\mathcal{I}_{\left\{k_{1}, \cdots, k_{N-1}\right\}}^{\left\{n_{1}, \cdots, n_{N-1}\right\}} / Z_{\mathrm{D} 6-\mathrm{D} 4^{\prime}}^{\left\{n_{1}, \cdots, n_{N-1}\right\}}$ for nonminuscule representations, preventing (3.17) from being true. This is because the elliptic genera of D2-branes receive contributions not only from the Higgs branch, in which D2-branes realize 6d self-dual strings, but also from the twisted Higgs branch, where D2branes become separable on $\mathrm{D} 4^{\prime}$-branes and move along the $x^{6}, x^{7}, x^{8}$ directions. Specifically, as D2-branes are away from NS5-branes, the latter configuration is no longer associated to $6 \mathrm{~d}(2,0)$ SCFTs which describe the worldvolume physics of NS5-branes. Instead, it corresponds to the monopole (string) bubbling sector for $5 \mathrm{~d}$ 't Hooft surface defects on D4'-branes wrapping $\mathbf{R}^{3} \times T^{2}$. For example, once we take the $M \rightarrow \epsilon_{+}$limit which Higgses D2-D6 multiplets, or equivalently removes the D6-brane, the elliptic genera $\mathcal{I}_{\left\{k_{1}, \cdots, k_{N-1}\right\}}^{\left\{n_{1}, \cdots, n_{N-1}\right\}} / Z_{\mathrm{D} 6-\mathrm{D} 4^{\prime}}^{\left\{n_{1}, \cdots, n_{N-1}\right\}}$ reduce to the elliptic uplift of the monopole bubbling indices in [52-54] where $\operatorname{sh}(x)$ is replaced with $\operatorname{sh}(x) \rightarrow \theta(x)$. 
Here we do not attempt to distinguish the Higgs branch states from the others as in section 2.3. We instead report the following observation: for most of the winding sectors $\left(k_{1}, \cdots, k_{N-1}\right)$, except those with non-trivial monopole bubbling indices in [52-54], the relation (3.17) holds true after discarding all the BPS states that carry non-trivial $\prod_{i=1}^{N-1} \mathrm{U}\left(n_{i}\right)$ flavor charges. More precisely, we take the series expansion of the right-hand side of (3.17) in the flavor fugacities $e^{-m_{\ell}^{(i)}}$ and compare its zero-th order coefficient with the left-hand side of (3.17). We found that

$$
\begin{aligned}
& \mathfrak{n}_{1}^{1} \cdot\left(\mathcal{W}_{\mathbf{3}}^{\mathrm{U}(2)}+\mathcal{W}_{\mathbf{1}}^{\mathrm{U}(2)}\right) / \mathcal{W}_{1}^{\mathrm{U}(2)}=Z_{6 d / 2 d}^{\{2\}} /\left.Z_{6 d / 2 d}^{\{0\}}\right|_{0 \text {-th }} \text { at } q^{0,1,2} \text { and } \mathfrak{n}_{1}^{2,3} \text { orders } \\
& \mathfrak{n}_{1}^{3 / 2} \cdot\left(\mathcal{W}_{\mathbf{4}}^{\mathrm{U}(2)}+2 \mathcal{W}_{\mathbf{2}}^{\mathrm{U}(2)}\right) / \mathcal{W}_{1}^{\mathrm{U}(2)}=Z_{6 d / 2 d}^{\{3\}} /\left.Z_{6 d / 2 d}^{\{0\}}\right|_{0 \text {-th }} \text { at } q^{0,1} \text { and } \mathfrak{n}_{1}^{3} \text { orders } \\
& \mathfrak{n}_{1}^{2 / 3} \mathfrak{n}_{2}^{4 / 3} \cdot\left(\mathcal{W}_{\mathbf{6}}^{\mathrm{U}(3)}+\mathcal{W}_{\overline{\mathbf{3}}}^{\mathrm{U}(3)}\right) / \mathcal{W}_{1}^{\mathrm{U}(3)}=Z_{6 d / 2 d}^{\{0,2\}} /\left.Z_{6 d / 2 d}^{\{0,0\}}\right|_{0 \text {-th }} \text { at } q^{0,1} \text { and } \mathfrak{n}_{1}^{1,2,3}, \mathfrak{n}_{2}^{2,3}, \mathfrak{n}_{1}^{2} \mathfrak{n}_{2}^{1}, \mathfrak{n}_{1}^{2} \mathfrak{n}_{2}^{2} \text { orders } \\
& \mathfrak{n}_{1}^{4 / 3} \mathfrak{n}_{2}^{2 / 3} \cdot\left(\mathcal{W}_{\overline{\mathbf{6}}}^{\mathrm{U}(3)}+\mathcal{W}_{\mathbf{3}}^{\mathrm{U}(3)}\right) / \mathcal{W}_{1}^{\mathrm{U}(3)}=Z_{6 d / 2 d}^{\{2,0\}} /\left.Z_{6 d / 2 d}^{\{0,0\}}\right|_{0 \text {-th }} \quad \text { at } q^{0,1} \text { and } \mathfrak{n}_{1}^{2,3}, \mathfrak{n}_{2}^{1,2,3}, \mathfrak{n}_{1}^{1} \mathfrak{n}_{2}^{2}, \mathfrak{n}_{1}^{2} \mathfrak{n}_{2}^{2} \text { orders } \\
& \mathfrak{n}_{1}^{1} \mathfrak{n}_{2}^{1} \cdot\left(\mathcal{W}_{\mathbf{8}}^{\mathrm{U}(3)}+\mathcal{W}_{1}^{\mathrm{U}(3)}\right) / \mathcal{W}_{1}^{\mathrm{U}(3)}=Z_{6 d / 2 d}^{\{1,1\}} /\left.Z_{6 d / 2 d}^{\{0,0\}}\right|_{0 \text {-th }} \quad \text { at } q^{0,1} \text { and } \mathfrak{n}_{1}^{1,2,3}, \mathfrak{n}_{2}^{1,2,3}, \mathfrak{n}_{1}^{1} \mathfrak{n}_{2}^{2}, \mathfrak{n}_{1}^{2} \mathfrak{n}_{2}^{1}, \mathfrak{n}_{1}^{2} \mathfrak{n}_{2}^{2} \text { orders. }
\end{aligned}
$$

For those exceptional sectors, the same truncation makes (3.17) true only at $q^{0}$ order, i.e.,

$$
\begin{aligned}
\mathfrak{n}_{1}^{1} \cdot\left(\mathcal{W}_{\mathbf{3}}^{\mathrm{U}(2)}+\mathcal{W}_{\mathbf{1}}^{\mathrm{U}(2)}\right) / \mathcal{W}_{\mathbf{1}}^{\mathrm{U}(2)} & =Z_{6 d / 2 d}^{\{2\}} /\left.Z_{6 d / 2 d}^{\{0\}}\right|_{0 \text {-th }} \\
\mathfrak{n}_{1}^{3 / 2} \cdot\left(\mathcal{W}_{\mathbf{4}}^{\mathrm{U}(2)}+2 \mathcal{W}_{\mathbf{2}}^{\mathrm{U}(2)}\right) / \mathcal{W}_{\mathbf{1}}^{\mathrm{U}(2)}=Z_{6 d / 2 d}^{\{3\}} /\left.Z_{6 d / 2 d}^{\{0\}}\right|_{0 \text {-th }} & \text { at } q^{0} \text { and } \mathfrak{n}_{1}^{1,2} \text { orders } \\
\mathfrak{n}_{1}^{2 / 3} \mathfrak{n}_{2}^{4 / 3} \cdot\left(\mathcal{W}_{\mathbf{6}}^{\mathrm{U}(3)}+\mathcal{W}_{\overline{\mathbf{3}}}^{\mathrm{U}(3)}\right) / \mathcal{W}_{\mathbf{1}}^{\mathrm{U}(3)}=Z_{6 d / 2 d}^{\{0,2\}} /\left.Z_{6 d / 2 d}^{\{0,0\}}\right|_{0 \text {-th }} & \text { at } q^{0} \text { and } \mathfrak{n}_{2}^{1}, \mathfrak{n}_{1}^{1} \mathfrak{n}_{2}^{1}, \mathfrak{n}_{1}^{1} \mathfrak{n}_{2}^{2} \text { orders } \\
\mathfrak{n}_{1}^{4 / 3} \mathfrak{n}_{2}^{2 / 3} \cdot\left(\mathcal{W}_{\overline{\mathbf{6}}}^{\mathrm{U}(3)}+\mathcal{W}_{\mathbf{3}}^{\mathrm{U}(3)}\right) / \mathcal{W}_{\mathbf{1}}^{\mathrm{U}(3)}=Z_{6 d / 2 d}^{\{2,0\}} /\left.Z_{6 d / 2 d}^{\{0,0\}}\right|_{0 \text {-th }} & \text { at } q^{0} \text { and } \mathfrak{n}_{1}^{1}, \mathfrak{n}_{1}^{1} \mathfrak{n}_{2}^{1}, \mathfrak{n}_{1}^{2} \mathfrak{n}_{2}^{1} \text { orders } \\
\mathfrak{n}_{1}^{1} \mathfrak{n}_{2}^{1} \cdot\left(\mathcal{W}_{\mathbf{8}}^{\mathrm{U}(3)}+\mathcal{W}_{\mathbf{1}}^{\mathrm{U}(3)}\right) / \mathcal{W}_{\mathbf{1}}^{\mathrm{U}(3)}=Z_{6 d / 2 d}^{\{1,1\}} /\left.Z_{6 d / 2 d}^{\{0,0\}}\right|_{0 \text {-th }} & \text { at } q^{0} \text { and } \mathfrak{n}_{1}^{1} \mathfrak{n}_{2}^{1} \text { order. }
\end{aligned}
$$

In spite of the extra D4' states present in $\mathcal{I}_{\left\{k_{1}, \cdots, k_{N-1}\right\}}^{\left\{n_{1}, \cdots, n_{N-1}\right\}} / Z_{\mathrm{D} 6-\mathrm{D} 4^{\prime}}^{\left\{n_{1}, \cdots, n_{N-1}\right\}}$, we still regard the above results (3.18)-(3.22) as a supporting evidence for the validity of the quiver gauge theory introduced in section 3.1. It would be desirable to come up with a way to precisely distinguish the 6d SCFT spectrum from the rest in the elliptic genera.

\subsection{1 qq-characters and $y$ observables}

As we did in section 2.2, let us conclude our discussion by adding a few remarks about the qq-character interpretation of our elliptic genus (3.13). Without D4' branes, the NS5-D6 systems we considered can be thought of as engineering a six-dimensional superconformal $\mathrm{U}(1)^{N-1}$ abelian $A_{N-1}$-quiver theory, i.e. a linear quiver theory with $N-1 \mathrm{U}(1)$ gauge nodes with bifundamental and/or fundamental matter. The qq-characters for such a theory, as studied in $[14,55,56]$, can be classified in terms of tensor product representations $\mathbf{R}$ of the $A_{N-1}$ Lie algebra. In our language they are constructed by adding one or more D $4^{\prime}$ branes to the NS5-D6 system, where the numbers $\left(n_{1}, \ldots, n_{N-1}\right)$ of $\mathrm{D} 4^{\prime}$ are related to the 
tensor product representation $\mathbf{R}=\otimes_{i=1}^{N-1}\left(\bigwedge^{N-i}\right)^{n_{i}}$ as in (3.16). More precisely we have

$$
\mathcal{I}_{0}^{-N} Z_{6 d / 2 d}^{\left\{n_{1}, \ldots, n_{N-1}\right\}} Z_{\mathrm{D} 6-\mathrm{D} 4^{\prime}}^{\left\{n_{1}, \cdots, n_{N-1}\right\}}=\sum_{k_{1}, \ldots, k_{N-1} \geqslant 0} \mathfrak{n}_{1}^{k_{1}} \ldots \mathfrak{n}_{N-1}^{k_{N-1}} \cdot \mathcal{I}_{\left\{k_{1}, \cdots, k_{N-1}\right\}}^{\left\{n_{1}, \cdots, n_{N-1}\right\}}=\mathcal{X}_{\mathbf{R}}^{\left(A_{N-1}\right)}\left(m_{\ell}^{(i)}\right) .
$$

Similarly to what happens in the five-dimensional case, the qq-characters for our sixdimensional quiver theory can be written in terms of correlators of $y_{i}$-operators, although in our setup we have a different $y_{i}$ for each abelian gauge node $(i=1, \ldots, N-1)$. Any correlator involving a number $l_{i}$ of $y_{i}$-observables (or their inverse) depending on mass parameters $x_{j}^{(i)}$ for each gauge node can be computed via

$$
\prod_{i=1}^{N-1} \prod_{j=1}^{l_{i}} y_{i}^{s_{j}^{(i)}}\left(x_{j}^{(i)}\right)=\sum_{k_{1}, \ldots, k_{N-1} \geqslant 0} \frac{\mathfrak{n}_{1}^{k_{1}} \ldots \mathfrak{n}_{N-1}^{k_{N-1}}}{k_{1} ! \ldots k_{N-1} !} \oint\left[\prod_{i=1}^{N-1} \prod_{s=1}^{k_{i}} \frac{d \phi_{s}^{(i)}}{2 \pi i}\right] Z_{k_{1} \ldots k_{N-1}}^{\mathrm{NSS}} Z_{k_{1} \ldots k_{N-1}}^{\mathrm{D} 6} \prod_{i=1}^{N-1} \prod_{j=1}^{l_{i}}\left[Z_{k_{i}}^{\mathrm{D} 4^{\prime}}\left(x_{j}^{(i)}\right)\right]^{s_{j}^{(i)}}
$$

with $s_{j}^{(i)}= \pm 1$ and where the functions appearing in the integrand were introduced in (3.9). For brevity, we suppressed their dependence on $\epsilon_{ \pm}$and $m$. The integral is evaluated by summing over the residues at the poles labeled by $(N-1)$-tuples of the Young tableaux (3.10); this pole prescription therefore differentiates correlators of $y_{i}$-operators from qq-characters, since the latter receive contributions also from poles of the form (3.11).

To illustrate the relation between $X$ and $y_{i}$ 's, let us consider a few examples. When $N=$ 2 the fundamental (i.e. $n_{1}=1$ ) qq-character, associated to the fundamental representation $\mathbf{2}$ of $A_{1}$, can be written as

$$
x_{\mathbf{2}}^{\left(A_{1}\right)}\left(m_{1}^{(1)}\right)=y_{1}\left(m_{1}^{(1)}\right)+\mathfrak{n}_{1} \frac{P\left(m_{1}^{(1)}\right)}{y_{1}\left(m_{1}^{(1)}+2 \epsilon_{+}\right)},
$$

where

$$
P(\sigma)=\frac{\theta\left(\sigma+m+\epsilon_{+}\right)}{\eta} \frac{\theta\left(\sigma-m+\epsilon_{+}\right)}{\eta} .
$$

The first higher qq-character $\left(n_{1}=2\right)$, corresponding to the representation $\mathbf{2} \otimes \mathbf{2}=\mathbf{3} \oplus \mathbf{1}$ of $A_{1}$, reads

$$
\begin{aligned}
& x_{\mathbf{3} \oplus \mathbf{1}}^{\left(A_{1}\right)}\left(m_{1}^{(1)}, m_{2}^{(1)}\right)= \\
& y_{1}\left(m_{1}^{(1)}\right) y_{1}\left(m_{2}^{(1)}\right)+\mathfrak{n}_{1} P\left(m_{1}^{(1)}\right) S\left(m_{1}^{(1)}-m_{2}^{(1)}\right) \frac{y_{1}\left(m_{2}^{(1)}\right)}{y_{1}\left(m_{1}^{(1)}+2 \epsilon_{+}\right)} \\
& \quad+\mathfrak{n}_{1} P\left(m_{2}^{(1)}\right) S\left(m_{2}^{(1)}-m_{1}^{(1)}\right) \frac{y_{1}\left(m_{1}^{(1)}\right)}{y_{1}\left(m_{2}^{(1)}+2 \epsilon_{+}\right)}+\mathfrak{n}_{1}^{2} P\left(m_{1}^{(1)}\right) P\left(m_{2}^{(1)}\right) \frac{1}{y_{1}\left(m_{1}^{(1)}+2 \epsilon_{+}\right) y_{1}\left(m_{2}^{(1)}+2 \epsilon_{+}\right)},
\end{aligned}
$$

with

$$
S(\sigma)=\frac{\theta\left(\sigma+\epsilon_{1}\right) \theta\left(\sigma+\epsilon_{2}\right)}{\theta(\sigma) \theta\left(\sigma+2 \epsilon_{+}\right)} .
$$


When $N=3$ the fundamental $\left(n_{1}=0, n_{2}=1\right)$ and antisymmetric $\left(n_{1}=1, n_{2}=0\right)$ qqcharacters, associated to the $A_{2}$ representations $\mathbf{3}$ and $\overline{\mathbf{3}}$ respectively, can be decomposed as

$$
\begin{aligned}
& x_{\mathbf{3}}^{\left(A_{2}\right)}\left(m_{1}^{(1)}\right)=y_{1}\left(m_{1}^{(1)}\right)+\mathfrak{n}_{1} P_{1}\left(m_{1}^{(1)}\right) \frac{y_{2}\left(m_{1}^{(1)}+\epsilon_{+}\right)}{y_{1}\left(m_{1}^{(1)}+2 \epsilon_{+}\right)}+\mathfrak{n}_{1} \mathfrak{n}_{2} \frac{P_{1}\left(m_{1}^{(1)}\right) P_{2}\left(m_{1}^{(1)}+\epsilon_{+}\right)}{y_{2}\left(m_{1}^{(1)}+3 \epsilon_{+}\right)}, \\
& x_{\overline{\mathbf{3}}}^{\left(A_{2}\right)}\left(m_{1}^{(2)}\right)=y_{2}\left(m_{1}^{(2)}\right)+\mathfrak{n}_{2} P_{2}\left(m_{1}^{(2)}\right) \frac{y_{1}\left(m_{1}^{(2)}+\epsilon_{+}\right)}{y_{2}\left(m_{1}^{(2)}+2 \epsilon_{+}\right)}+\mathfrak{n}_{1} \mathfrak{n}_{2} \frac{P_{2}\left(m_{1}^{(2)}\right) P_{1}\left(m_{1}^{(2)}+\epsilon_{+}\right)}{y_{1}\left(m_{1}^{(2)}+3 \epsilon_{+}\right)},
\end{aligned}
$$

with

$$
P_{1}(\sigma)=\frac{\theta\left(\sigma+m+\epsilon_{+}\right)}{\eta}, \quad P_{2}(\sigma)=\frac{\theta\left(\sigma-2 m+\epsilon_{+}\right)}{\eta} .
$$

Similar relations can be found for any $N$ and any representation $\mathbf{R}=\otimes_{i=1}^{N-1}\left(\bigwedge^{N-i}\right)^{n_{i}}[56]$.

\section{Concluding remarks}

In this paper we studied the $5 \mathrm{~d} \mathcal{N}=1^{*}$ Wilson loops in a generic $\mathrm{U}(N)$ gauge representation, using the ADHM quantum mechanics of D0-D4-D4' branes. The partition function for the grand canonical ensemble of D0-branes is the sum over products between two different Wilson loops, supported on D4 and D4'-branes respectively. We illustrated how to disentangle individual Wilson loops from the full D0-brane partition function in a number of examples. They can be interpreted as the Wilson surface observables in M5-branes, based on the correspondence between 5d maximal U(N) SYM and 6d $(2,0)$ SCFT of $A_{N-1^{-}}$ type. We also studied the Wilson surfaces using the $2 \mathrm{~d}(0,4)$ quiver gauge theories, which correspond to the UV description of self-dual strings in the presence of a Wilson surface defect. Both computations are in agreement for the minuscule Wilson surfaces. On the other hand, the elliptic genera for the non-minuscule representations capture extra BPS states irrelevant from $6 \mathrm{~d}(2,0)$ SCFT, displaying only a partial agreement between two independent computations.

There are a number of open questions that deserve further investigation. It would be important to understand how to distinguish a contribution from extra BPS states, captured in the elliptic genera, supported on D4'-branes. It would also be a primary step to study the Wilson surface observables in $(1,0)$ SCFTs, such as E-string theory, in which an emergence of extra states in the elliptic genera is generically observed. Finally, it may be interesting to make a parallel study on the codimension-2 defects in M5-branes, either from 5d SYM perspective or from $6 \mathrm{~d}$ self-dual strings' perspective.

\section{Acknowledgments}

We thank Dongmin Gang, Dongwook Ghim, Chiung Hwang, Sungjay Lee, Piljin Yi, Youngbin Yun, and especially Benjamin Assel, Hee-Cheol Kim, Kimyeong Lee for helpful discussions and comments. PA and SK are supported in part by the National Research Foundation of Korea (NRF) Grant 2015R1A2A2A01003124 and 2018R1A2B6004914. The work of 
PA is also supported in part by Samsung Science and Technology Foundation under Project Number SSTF-BA1402-08 and by the Korea Research Fellowship Program through the National Research Foundation of Korea funded by the Ministry of Science, ICT and Future Planning, grant number 2016H1D3A1938054.

Open Access. This article is distributed under the terms of the Creative Commons Attribution License (CC-BY 4.0), which permits any use, distribution and reproduction in any medium, provided the original author(s) and source are credited.

\section{References}

[1] O.J. Ganor, Six-dimensional tensionless strings in the large $N$ limit, Nucl. Phys. B 489 (1997) 95 [hep-th/9605201] [INSPIRE].

[2] B. Chen, W. He, J.-B. Wu and L. Zhang, M5-branes and Wilson Surfaces, JHEP 08 (2007) 067 [arXiv: 0707.3978] [INSPIRE].

[3] E. Witten, Some comments on string dynamics, in proceedings of the STRINGS 95: Future Perspectives in String Theory, Los Angeles, California, U.S.A., 13-18 March 1995, pp. 501-523 [hep-th/9507121] [INSPIRE].

[4] A. Strominger, Open p-branes, Phys. Lett. B 383 (1996) 44 [hep-th/9512059] [INSPIRE].

[5] J.M. Maldacena, Wilson loops in large $N$ field theories, Phys. Rev. Lett. 80 (1998) 4859 [hep-th/9803002] [INSPIRE].

[6] D.E. Berenstein, R. Corrado, W. Fischler and J.M. Maldacena, The Operator product expansion for Wilson loops and surfaces in the large N limit, Phys. Rev. D 59 (1999) 105023 [hep-th/9809188] [INSPIRE].

[7] R. Corrado, B. Florea and R. McNees, Correlation functions of operators and Wilson surfaces in the $d=6,(0,2)$ theory in the large $N$ limit, Phys. Rev. D 60 (1999) 085011 [hep-th/9902153] [INSPIRE].

[8] O. Lunin, 1/2-BPS states in M-theory and defects in the dual CFTs, JHEP 10 (2007) 014 [arXiv: 0704.3442] [INSPIRE].

[9] B. Chen, C.-Y. Liu and J.-B. Wu, Operator Product Expansion of Wilson surfaces from M5-branes, JHEP 01 (2008) 007 [arXiv:0711.2194] [INSPIRE].

[10] B. Chen and J.-B. Wu, Wilson-Polyakov surfaces and M-theory branes, JHEP 05 (2008) 046 [arXiv: 0802.2173] [INSPIRE].

[11] H.-C. Kim, J. Kim and S. Kim, Instantons on the 5-sphere and M5-branes, arXiv: 1211.0144 [INSPIRE].

[12] J.A. Minahan, A. Nedelin and M. Zabzine, 5D super Yang-Mills theory and the correspondence to $A d S_{7} / C F T_{6}$, J. Phys. A 46 (2013) 355401 [arXiv:1304.1016] [INSPIRE].

[13] M. Bullimore and H.-C. Kim, The Superconformal Index of the $(2,0)$ Theory with Defects, JHEP 05 (2015) 048 [arXiv: 1412.3872] [INSPIRE].

[14] N. Nekrasov, BPS/CFT correspondence: non-perturbative Dyson-Schwinger equations and qq-characters, JHEP 03 (2016) 181 [arXiv:1512.05388] [INSPIRE].

[15] H.-C. Kim, Line defects and 5d instanton partition functions, JHEP 03 (2016) 199 [arXiv: 1601.06841] [INSPIRE]. 
[16] H. Mori and S. Yamaguchi, M5-branes and Wilson surfaces in $A d S_{7} / C F T_{6}$ correspondence, Phys. Rev. D 90 (2014) 026005 [arXiv: 1404.0930] [InSPIRE].

[17] D. Tong and K. Wong, Instantons, Wilson lines and D-branes, Phys. Rev. D 91 (2015) 026007 [arXiv: 1410.8523 ] [INSPIRE].

[18] B. Haghighat, A. Iqbal, C. Kozçaz, G. Lockhart and C. Vafa, M-Strings, Commun. Math. Phys. 334 (2015) 779 [arXiv:1305.6322] [InSPIRE].

[19] B. Haghighat, C. Kozcaz, G. Lockhart and C. Vafa, Orbifolds of M-strings, Phys. Rev. D 89 (2014) 046003 [arXiv:1310.1185] [INSPIRE].

[20] J. Gomis and F. Passerini, Holographic Wilson Loops, JHEP 08 (2006) 074 [hep-th/0604007] [INSPIRE].

[21] B. Assel and J. Gomis, Mirror Symmetry And Loop Operators, JHEP 11 (2015) 055 [arXiv: 1506.01718] [INSPIRE].

[22] N.S. Manton, A Remark on the Scattering of BPS Monopoles, Phys. Lett. B 110 (1982) 54 [INSPIRE].

[23] M.R. Douglas, Branes within branes, in proceedings of the NATO Advanced Study Institute on Strings, Branes and Dualities, Cargèse, France, 26 May-14 June 1997, pp. 267-275 [hep-th/9512077] [INSPIRE].

[24] D. Tong, The holographic dual of $A d S_{3} \times S^{3} \times S^{3} \times S^{1}$, JHEP 04 (2014) 193 [arXiv: 1402.5135] [INSPIRE].

[25] E. Witten, $\sigma$-models and the ADHM construction of instantons, J. Geom. Phys. 15 (1995) 215 [hep-th/9410052] [INSPIRE].

[26] N.A. Nekrasov, Seiberg-Witten prepotential from instanton counting, Adv. Theor. Math. Phys. 7 (2003) 831 [hep-th/0206161] [INSPIRE].

[27] N. Nekrasov and A. Okounkov, Seiberg-Witten theory and random partitions, Prog. Math. 244 (2006) 525 [hep-th/0306238] [INSPIRE].

[28] C. Hwang, J. Kim, S. Kim and J. Park, General instanton counting and 5d SCFT, JHEP 07 (2015) 063 [arXiv: 1406.6793] [InSPIRE].

[29] K. Hori, H. Kim and P. Yi, Witten Index and Wall Crossing, JHEP 01 (2015) 124 [arXiv: 1407.2567] [INSPIRE].

[30] C. Cordova and S.-H. Shao, An Index Formula for Supersymmetric Quantum Mechanics, arXiv: 1406.7853 [INSPIRE].

[31] R. Flume and R. Poghossian, An Algorithm for the microscopic evaluation of the coefficients of the Seiberg-Witten prepotential, Int. J. Mod. Phys. A 18 (2003) 2541 [hep-th/0208176] [INSPIRE].

[32] U. Bruzzo, F. Fucito, J.F. Morales and A. Tanzini, Multiinstanton calculus and equivariant cohomology, JHEP 05 (2003) 054 [hep-th/0211108] [INSPIRE].

[33] A. Iqbal, C. Kozcaz and K. Shabbir, Refined Topological Vertex, Cylindric Partitions and the U(1) Adjoint Theory, Nucl. Phys. B 838 (2010) 422 [arXiv:0803.2260] [inSPIRE].

[34] R. Poghossian and M. Samsonyan, Instantons and the 5D U(1) gauge theory with extra adjoint, J. Phys. A 42 (2009) 304024 [arXiv:0804.3564] [INSPIRE]. 
[35] H.-C. Kim, S. Kim, E. Koh, K. Lee and S. Lee, On instantons as Kaluza-Klein modes of M5-branes, JHEP 12 (2011) 031 [arXiv:1110.2175] [INSPIRE].

[36] M. Del Zotto and G. Lockhart, On Exceptional Instanton Strings, JHEP 09 (2017) 081 [arXiv: 1609.00310] [INSPIRE].

[37] M. Del Zotto, J. Gu, M.-X. Huang, A.-K. Kashani-Poor, A. Klemm and G. Lockhart, Topological Strings on Singular Elliptic Calabi-Yau 3-folds and Minimal 6d SCFTs, JHEP 03 (2018) 156 [arXiv:1712.07017] [INSPIRE].

[38] J. Kim, K. Lee and J. Park, On elliptic genera of 6d string theories, arXiv:1801.01631 [INSPIRE].

[39] S. Shadchin, Saddle point equations in Seiberg-Witten theory, JHEP 10 (2004) 033 [hep-th/0408066] [INSPIRE].

[40] A. Hanany and E. Witten, Type IIB superstrings, BPS monopoles and three-dimensional gauge dynamics, Nucl. Phys. B 492 (1997) 152 [hep-th/9611230] [InSPIRE].

[41] E. Witten, Phases of $N=2$ theories in two-dimensions, Nucl. Phys. B 403 (1993) 159 [hep-th/9301042] [INSPIRE].

[42] M. Bertolini, I.V. Melnikov and M.R. Plesser, Accidents in $(0,2)$ Landau-Ginzburg theories, JHEP 12 (2014) 157 [arXiv: 1405.4266] [INSPIRE].

[43] A. Gadde and P. Putrov, Exact solutions of $(0,2)$ Landau-Ginzburg models, arXiv: 1608.07753 [INSPIRE].

[44] A. Gadde and S. Gukov, 2d Index and Surface operators, JHEP 03 (2014) 080 [arXiv: 1305.0266] [INSPIRE].

[45] F. Benini, R. Eager, K. Hori and Y. Tachikawa, Elliptic genera of two-dimensional $N=2$ gauge theories with rank-one gauge groups, Lett. Math. Phys. 104 (2014) 465 [arXiv: 1305.0533] [INSPIRE].

[46] F. Benini, R. Eager, K. Hori and Y. Tachikawa, Elliptic Genera of $2 d \mathcal{N}=2$ Gauge Theories, Commun. Math. Phys. 333 (2015) 1241 [arXiv: 1308.4896] [INSPIRE].

[47] N. Nekrasov and V. Pestun, Seiberg-Witten geometry of four dimensional $N=2$ quiver gauge theories, arXiv:1211.2240 [INSPIRE].

[48] A. Gadde, B. Haghighat, J. Kim, S. Kim, G. Lockhart and C. Vafa, 6 d String Chains, JHEP 02 (2018) 143 [arXiv: 1504.04614] [INSPIRE].

[49] M.R. Douglas, On D = 5 super Yang-Mills theory and (2,0) theory, JHEP 02 (2011) 011 [arXiv: 1012.2880] [INSPIRE].

[50] N. Lambert, C. Papageorgakis and M. Schmidt-Sommerfeld, M5-Branes, D4-branes and Quantum 5D super-Yang-Mills, JHEP 01 (2011) 083 [arXiv:1012.2882] [INSPIRE].

[51] C. Cordova, T.T. Dumitrescu and X. Yin, Higher Derivative Terms, Toroidal Compactification and Weyl Anomalies in Six-Dimensional $(2,0)$ Theories, arXiv: 1505.03850 [INSPIRE].

[52] Y. Ito, T. Okuda and M. Taki, Line operators on $S^{1} \times \mathbb{R}^{3}$ and quantization of the Hitchin moduli space, JHEP 04 (2012) 010 [Erratum JHEP 03 (2016) 085] [arXiv:1111.4221] [INSPIRE].

[53] N. Mekareeya and D. Rodriguez-Gomez, $5 d$ gauge theories on orbifolds and $4 d$ 't Hooft line indices, JHEP 11 (2013) 157 [arXiv:1309.1213] [INSPIRE]. 
[54] T.D. Brennan, A. Dey and G.W. Moore, On 't Hooft Defects, Monopole Bubbling and Supersymmetric Quantum Mechanics, arXiv:1801.01986 [INSPIRE].

[55] T. Kimura and V. Pestun, Quiver elliptic W-algebras, Lett. Math. Phys. 108 (2018) 1383 [arXiv: 1608.04651] [INSPIRE].

[56] T. Kimura, H. Mori and Y. Sugimoto, Refined geometric transition and qq-characters, JHEP 01 (2018) 025 [arXiv: 1705.03467] [INSPIRE]. 